\title{
Adaptive Neural Back-Stepping Control with Constrains for a Flexible Air-Breathing Hypersonic Vehicle
}

\author{
Pengfei Wang, Jie Wang, Jianming Shi, Chang Luo, Shili Tan, and Qiyun Xu \\ Air and Missile Defense College, Air Force Engineering University, Xian 710051, China \\ Correspondence should be addressed to Pengfei Wang; wang5899229@126.com
}

Received 17 May 2015; Revised 25 October 2015; Accepted 8 November 2015

Academic Editor: Xinggang Yan

Copyright ( 2015 Pengfei Wang et al. This is an open access article distributed under the Creative Commons Attribution License, which permits unrestricted use, distribution, and reproduction in any medium, provided the original work is properly cited.

\begin{abstract}
The design of an adaptive neural back-stepping control for a flexible air-breathing hypersonic vehicle (AHV) in the presence of input constraint and aerodynamic uncertainty is discussed. Based on functional decomposition, the dynamics can be decomposed into the velocity subsystem and the altitude subsystem. To guarantee the exploited controller's robustness with respect to parametric uncertainties, neural network (NN) is applied to approximate the lumped uncertainty of each subsystem of AHV model. The exceptional contribution is that novel auxiliary systems are introduced to compensate both the tracking errors and desired control laws, based on which the explored controller can still provide effective tracking of velocity and altitude commands when the actuators are saturated. Finally, simulation studies are made to illustrate the effectiveness of the proposed control approach in spite of the flexible effects, system uncertainties, and varying disturbances.
\end{abstract}

\section{Introduction}

Air-breathing hypersonic vehicles (AHV) are crucial because they may represent a more efficient way to make access to space routine or even make the space travel routine and intercontinental travel as easy as intercity travel. A key issue in making AHV feasible and efficient is the flight control design [1]. However, the flight control of AHV is still an open challenge due to its peculiarity of flight dynamics. It is worth noting that there exists strong coupling between the propulsive and the aerodynamic forces, which makes the aerodynamic characteristics of AHV very difficult to be estimated and measured [2,3].

Recently, lots of efforts have been put into flight control for AHV. Linear control theory was widely employed for flight control design based on linearized model. As shown in [4], the robust control of AHV is studied by introducing a linear quadratic regulator (LQR) with stochastic robustness analysis. In [5], LQR controller is proposed for the linearization model based on any equilibrium points of flight envelope.

Nonlinear control scheme is also employed for AHV. Back-stepping has been proved to be a powerful tool for the tracking control of a large class of strict-feedback systems or pure-feedback ones [6-12]. Back-stepping design for nonlinear systems consists of a recursive design procedure, which breaks down the full system control problem into a sequence of designs for lower-order subsystems. A back-stepping controller is designed with multilayer online adaptive neural networks, which can provide good tracking performance [13]. In [14], a combination of novelty command filtered back-stepping technology and dynamic inversion methodology is adopted for designing a dynamic state-feedback controller that provides stable tracking of the altitude and velocity reference commands. However, it is well known that there exists a problem of "explosion of terms" in the traditional back-stepping design, which is caused by the repeated differentiations of virtual control laws. To solve this problem, dynamic surface control [15] and tracking differentiators [16] should be applied.

Since the aerodynamic characteristics of AHV are sensitive to the flight condition changes, they are difficult to be measured. Thus, the aerodynamic uncertainty needs to be dealt with at the control design as the main issues. Conventionally, the flight control is accomplished by feedback linearization with neural networks to deal with the uncertainties. Efforts in [17] approximate the unknown nonlinear functions by radial basis function networks and incorporating the dynamic surface technique into a neural network 
based adaptive control design framework. Reference [18] investigates the discrete time controller for the longitudinal dynamics of the hypersonic flight vehicle with throttle setting constraint. The controller is proposed by estimating the system uncertainty and unknown control gain separately with neural networks. The auxiliary error signal is designed to compensate the effect of throttle setting constraint.

Moreover, input constraint also cannot be ignored in practice since the outputs of actuators are constrained for physical limitations. If the input constraint is ignored, control systems may suffer from performance limitations or even lose stability [19-21]. Much literature theoretically focuses on the control problem with input constraint [2226]. In practice, when input constraint occurs, aircraft body may change seriously even disintegrating. So it is necessary to research the control design problem with input constraint.

Motivated by the results of the previous studies, an adaptive neural back-stepping control approach is addressed for the longitudinal dynamical model of AHV. By viewing the flexible effects as system uncertainties, the longitudinal dynamics of AHV are decomposed into two functional subsystems, namely, the respective velocity subsystem and altitude subsystem. To ensure the controller's robustness, NNs are applied to estimate the lumped uncertainty of each subsystem. Particularly, novel auxiliary systems are exploited to deal with the problem of control input constraint. Finally, simulation results are presented to demonstrate the efficacy of the proposed control methodology. The special advantages of the approach proposed herein include the following:

(1) The novel auxiliary systems are employed to eliminate the error between the desired control laws and actual control laws, which makes sure that the semiglobally uniformly bounded stability of closed-loop system can be still achieved even when the physical limitations are in effect.

(2) The second-order reference model is designed for the precise estimation of the derivatives of virtual control laws, which predigests the design of controller.

The organization of paper is outlined as follows. Firstly, the longitudinal motion model and control-oriented model of an AHV are described in Section 2. Section 3 presents the design procedures of adaptive neural backstepping controller. Then, simulation results are given in Section 4. Finally, brief concluding remarks end the paper in Section 5 .

\section{Problem Formulation}

2.1. Longitudinal Dynamic Model of AHV. The model taken into consideration in this paper is developed by Bolender and Doman [27] for the longitudinal dynamics of an AHV. The flexible effects are included in these equations by modeling the vehicle as a single flexible structure with mass-normalized mode shapes.
Assuming a flat Earth and normalizing the span of an AHV to unit depth, the nonlinear motion equations are written as [27]

$$
\begin{aligned}
& \dot{V}=\frac{T \cos \alpha-D}{m}-\frac{\mu \sin \gamma}{\left(R_{E}+h\right)^{2}}, \\
& \dot{h}=V \sin \gamma, \\
& \dot{\gamma}=\frac{L+T \sin \alpha}{m V}-\frac{\left[\mu-V^{2}\left(R_{E}+h\right)\right] \cos \gamma}{V\left(R_{E}+h\right)^{2}}, \\
& \dot{\alpha}=-\dot{\gamma}+Q, \\
& \dot{Q}=\frac{z_{T} T+M}{I_{y y}}, \\
& \ddot{\eta}_{i}=-2 \zeta_{i} \omega_{i} \dot{\eta}_{i}-\omega_{i}^{2} \eta_{i}+N_{i}, \quad i=1,2,3,
\end{aligned}
$$

where the rigid body states $V, h, \gamma, \theta$, and $Q$ represent velocity, altitude, flight path angle, pitch angle, and pitch rate, respectively; $\mu$ and $R_{E}$ are gravity constant and the radial distance from center of the earth; $m$ and $I_{y y}$ are mass of vehicle and moment of inertia about pitch axis; $T, D, L$, and $M$ represent thrust, drag, lift, and pitch moment, respectively; $z_{T}$ denotes the thrust moment arm; the flexible states $\boldsymbol{\eta}=$ $\left[\begin{array}{llllll}\eta_{1} & \dot{\eta}_{1} & \eta_{2} & \dot{\eta}_{2} & \eta_{3} & \dot{\eta}_{3}\end{array}\right]^{T}$ denote the first three bending modes of the fuselage; $0<\zeta_{i}<1$ and $\omega_{i}>0(i=1,2,3)$ mean the damping ratio and natural frequency of the mass-normalized generalized coordinates of the flexible structure.

The dynamic system of an AHV can be decomposed as velocity subsystem and altitude-related subsystem. Define the control inputs $u=\left[\Phi, \delta_{e}\right]$, which are fuel equivalence ratio and elevator angular deflection, respectively.

Remark 1. In [28], the controller design requires an auxiliary actuator as the canard. However, the presence of a canard is quite problematic for the vehicle configuration, as this control surface must withstand the expected high temperatures at hypersonic speed. Therefore, it is assumed that only the fuel-to-air ratio and elevator are the actuators available for controlling the vehicle.

Let

$$
\bar{L}=L+T \sin \alpha .
$$

The thrust $T$, drag $D$, lift $\bar{L}$, pitch moment $M$, and generalized forces $N_{i}(i=1,2,3)$ are defined as

$$
\begin{aligned}
& T=\bar{q} S C_{T}(\alpha, M a, \Phi)+\varepsilon_{T}(\mathbf{x}, \boldsymbol{\eta}, \mathbf{u}), \\
& D=\bar{q} S C_{D}\left(\alpha, M a, \delta_{e}\right)+\varepsilon_{D}(\mathbf{x}, \boldsymbol{\eta}, \mathbf{u}), \\
& \bar{L}=\bar{q} S C_{\bar{L}}(\alpha, M a, \Phi)+\varepsilon_{\bar{L}}(\mathbf{x}, \boldsymbol{\eta}, \mathbf{u}), \\
& M=\bar{q} S \bar{c} C_{M}\left(\alpha, M a, \delta_{e}\right)+\varepsilon_{M}(\mathbf{x}, \boldsymbol{\eta}, \mathbf{u}), \\
& C_{T}(\alpha, M a, \Phi)=C_{T}^{0}(\alpha, M a)+C_{T}^{\Phi}(\alpha, M a, \Phi) \Phi, \\
& C_{M}\left(\alpha, M a, \delta_{e}\right)=C_{M}^{0}(\alpha, M a)+C_{M}^{\delta_{e}}\left(\alpha, M a, \delta_{e}\right) \delta_{e},
\end{aligned}
$$




$$
\begin{aligned}
& C_{\bar{L}}(\alpha, M a, \Phi)=C_{\bar{L}}^{0}(M a, \Phi)+C_{\bar{L}}^{\alpha}(\alpha, M a, \Phi) \alpha, \\
& N_{i} \approx \bar{q} S\left[N_{i}^{\alpha^{2}} \alpha^{2}+N_{i}^{\alpha} \alpha+N_{i}^{\delta_{e}} \delta_{e}+N_{i}^{0}+N_{i}^{\eta} \boldsymbol{\eta}\right],
\end{aligned}
$$

where $\bar{q}, S$, and $\bar{c}$ stand for the respective dynamic pressure, reference area, and aerodynamic chord. $\varepsilon_{T}, \varepsilon_{\bar{L}}, \varepsilon_{D}$, and $\varepsilon_{M}$ are denoted as the fitting error of $T, \bar{L}, D$, and $M$, respectively.

To be convenient for design of back-stepping, (1) can be rewritten as

$$
\begin{aligned}
\dot{V}= & f_{V}\left(\bar{q}, M a, \alpha, \delta_{e}\right)+g_{V}(\bar{q}, \alpha, M a, \Phi) \Phi+F_{V} \\
& +\Delta_{V}(\mathbf{x}, \boldsymbol{\eta}, \mathbf{u}), \\
\dot{h}= & V \gamma+\Delta_{h}(V, \gamma), \\
\dot{\gamma}= & f_{\gamma}(\bar{q}, M a, \Phi)+g_{\gamma}(\bar{q}, \alpha, M a, \Phi) \alpha+F_{\gamma} \\
& +\Delta_{\gamma}(\mathbf{x}, \boldsymbol{\eta}, \mathbf{u}), \\
\dot{\alpha}= & f_{\alpha}(\bar{q}, \alpha, M a, \Phi)+Q+F_{\alpha}+\Delta_{\alpha}(\mathbf{x}, \boldsymbol{\eta}, \mathbf{u}), \\
\dot{Q}= & f_{Q}(\bar{q}, \alpha, M a, \Phi)+g_{Q}\left(\bar{q}, \alpha, M a, \Phi, \delta_{e}\right) \delta_{e} \\
& +\Delta_{Q}(\mathbf{x}, \boldsymbol{\eta}, \mathbf{u})
\end{aligned}
$$

with

$$
\begin{aligned}
& f_{V}=\frac{\bar{q} S\left(C_{T}^{0} \cos \alpha-C_{D}\right)}{m}, \\
& g_{V}=\bar{q} S C_{T}^{\Phi} \frac{\cos \alpha}{m} \\
& f_{\gamma}=\frac{\bar{q} S C_{L}^{0}}{(m V)} \\
& g_{\gamma}=\frac{\bar{q} S C_{L}^{\alpha}}{(m V)}, \\
& f_{\alpha}=-\left(f_{\gamma}+g_{\gamma} \alpha\right) \\
& f_{Q}=\frac{\bar{q} S\left[z_{T}\left(C_{T}^{0}+C_{T}^{\Phi} \Phi\right)+\bar{c} C_{M}^{0}\right]}{I_{y y}}, \\
& g_{Q}=\frac{\bar{q} S \bar{c} C_{M}^{\delta_{e}}}{I_{y y}}, \\
& F_{V}=-\frac{\mu \sin \gamma}{\left(R_{E}+h\right)^{2}}, \\
& \left.\left.F_{\gamma}=-\frac{\left[\mu-V^{2}\left(R_{E}+h\right)\right] \cos \gamma}{E} . h\right)^{2}\right]
\end{aligned}
$$

The model errors are considered as

$$
\begin{aligned}
\Delta_{V}(\mathbf{x}, \boldsymbol{\eta}, \mathbf{u}) & =\frac{\left(\varepsilon_{T} \cos \alpha-\varepsilon_{D}\right)}{m}, \\
\Delta_{h}(V, \gamma) & =\frac{V \bar{\gamma}^{2} \sin \bar{\gamma}}{2},
\end{aligned}
$$

$$
\begin{aligned}
\Delta_{\gamma}(\mathbf{x}, \boldsymbol{\eta}, \mathbf{u}) & =\frac{\varepsilon_{\bar{L}}}{m V}, \\
\Delta_{\alpha}(\mathbf{x}, \boldsymbol{\eta}, \mathbf{u}) & =-\frac{\varepsilon_{\bar{L}}}{m V}, \\
\Delta_{Q}(\mathbf{x}, \boldsymbol{\eta}, \mathbf{u}) & =\frac{\left(z_{T} \varepsilon_{T}+\varepsilon_{M}\right)}{I_{y y}} .
\end{aligned}
$$

Remark 2. The flexible dynamics are taken as perturbations on the rigid body system, and their effects are evaluated in simulation. According to (1) and (4), $\eta_{i}$ would be asymptotic stability if $\Phi, \delta_{e}$, and $\alpha$ are bounded.

2.2. Control Objective. In practice, due to physical limitations, the outputs of the actuator are constrained. Input constraints studied in the paper include the constraint on fuel equivalence ratio, elevator deflection. The constraint on fuel equivalence ratio is imposed by the very nature of the propulsion system, which is required to maintain the conditions that sustain scramjet operation [29]. If the limit is violated, the thermal choking will occur. It could induce that engine unstarts which could jeopardize mission, vehicle, and its contents [30]. The constraints on elevator deflection and canard deflection are mainly imposed by the limits on control surface displacement. The above input constraint can be expressed as

$$
\begin{aligned}
& \Phi=\Phi_{\max }, \Phi_{c} \geq \Phi_{\max }, \\
& \Phi=\Phi_{c}, \Phi_{\min } \leq \Phi_{c} \leq \Phi_{\max }, \\
& \Phi=\Phi_{\min }, \quad \Phi_{c} \leq \Phi_{\min }, \\
& \delta_{e}=\delta_{e_{\max }}, \quad \delta_{e_{c}} \geq \delta_{e \max }, \\
& \delta_{e}=\delta_{e c}, \delta_{e \min } \leq \delta_{e_{c}} \leq \delta_{e \max }, \\
& \delta_{e}=\delta_{e \min }, \delta_{e c} \leq \delta_{e \min },
\end{aligned}
$$

where $\Phi_{\max }$ and $\Phi_{\min }$ denote the upper and lower bound of $\Phi$, respectively; $\delta_{e \max }$ and $\delta_{e \min }$ stand for the upper and lower bound of $\delta_{e}$, respectively; $\Phi_{c}$ and $\delta_{e_{c}}$ are the desired control inputs to be designed in the subsequent section.

A second-order reference model with amplitude, rate, and bandwidth limitation is introduced to deal with the input constraint. The structure of second-order reference model is given in Figure 1. $\Phi^{0}, \delta_{e}^{0}$, and $\gamma_{c}^{0}, \alpha_{c}^{0}$, and $Q_{c}^{0}$ are the designed actual control variables and virtual control variables. Define $\Phi, \delta_{e}$, and $\gamma_{c}, \alpha_{c}$, and $Q_{c}$ as the executable control laws and virtual control laws with the second-order reference model.

2.3. Radial Basis Function NN Approximation. The radial basis function NN (RBFNN) will be introduced to approach the unknown functions $f_{x}(\mathbf{z})$ and $g_{x}(\mathbf{z})(x=V, \gamma, Q)$ owing to its excellent performance and global approximation. It has been proved that RBFNN can approximate an arbitrary continuous function over a compact set $\Omega_{\mathbf{z}} \subseteq \mathbf{R}^{n}$ to an arbitrary accuracy. RBFNN is formulated as $\boldsymbol{\theta}^{T} \boldsymbol{\xi}(\mathbf{z})$ with the input vector $\mathbf{z} \subseteq \mathbf{R}^{n}$, the weight vector $\boldsymbol{\theta} \subseteq \mathbf{R}^{m}$, the basis 


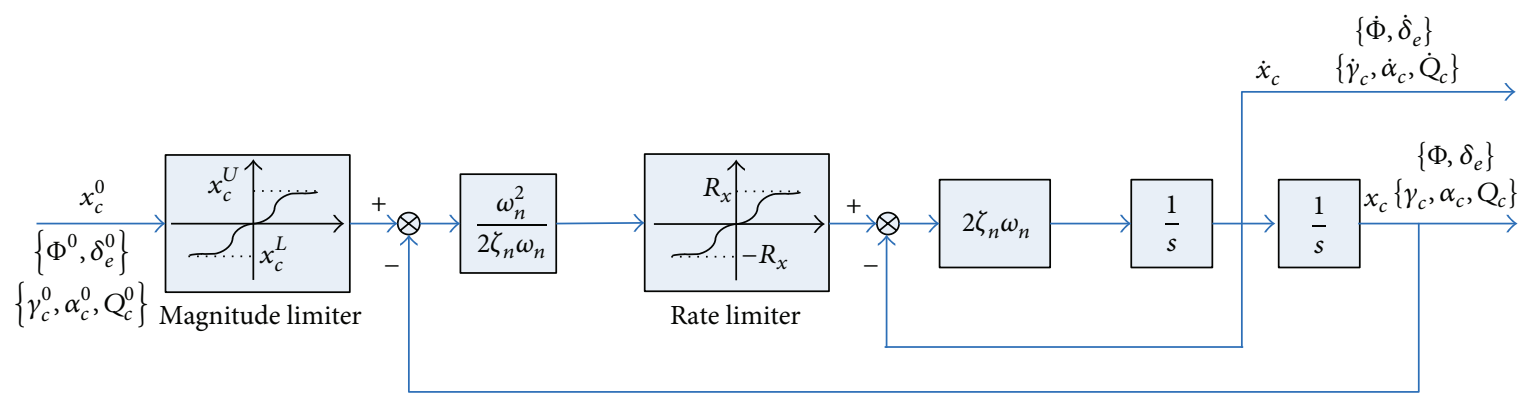

FIGURE 1: Second-order reference model.

function vector $\boldsymbol{\xi}(\mathbf{z}) \subseteq \mathbf{R}^{m}$, the node number $m$, and the input number $n$. The basis function is selected as the following Gaussian function:

$$
\xi_{j}(\mathbf{z})=\exp \left(-\frac{\left\|\mathbf{z}-\mathbf{c}_{i}\right\|^{2}}{2 b_{i}^{2}}\right), \quad i=1,2, \ldots, m
$$

where $\mathbf{c}_{i}=\left[c_{i 1}, c_{i 2}, \ldots, c_{i m}\right]^{T}$ and $b_{i}$ stand for the center and width of Gaussian function, respectively.

Assumption 3. $f_{x}(z)$ and $g_{x}(z)(x=V, \gamma, Q)$ are continuous real function. The unknown functions $f_{x}(\mathbf{z})$ and $g_{x}(\mathbf{z})$ can be designed as

$$
\begin{aligned}
& f_{x}(\mathbf{z})=\boldsymbol{\theta}_{f_{x}}^{* T} \boldsymbol{\xi}_{f_{x}}(\mathbf{z})+\varepsilon_{f_{x}}^{*}, \\
& g_{x}(\mathbf{z})=\boldsymbol{\theta}_{g_{x}}^{* T} \boldsymbol{\xi}_{g_{x}}(\mathbf{z})+\varepsilon_{g_{x}}^{*},
\end{aligned}
$$

where $\boldsymbol{\xi}_{f_{x}}(\mathbf{z})$ and $\boldsymbol{\xi}_{g_{x}}(\mathbf{z})$ are radial basis function vector; $\varepsilon_{f_{x}}^{*}$ and $\varepsilon_{g_{x}}^{*}$ denote the approximation error, and $\left|\varepsilon_{f_{x}}^{*}\right| \leq \varepsilon_{f_{x}}^{M}$ and $\left|\varepsilon_{g_{x}}^{*}\right| \leq \varepsilon_{g_{x}}^{M} ; \boldsymbol{\theta}_{f_{x}}^{*}$ and $\boldsymbol{\theta}_{g_{x}}^{*}$ represent optimal weight vector, $\widehat{\boldsymbol{\theta}}_{f_{x}}$ and $\widehat{\boldsymbol{\theta}}_{g_{x}}$ are the estimation of $\boldsymbol{\theta}_{f_{x}}^{*}$ and $\boldsymbol{\theta}_{g_{x}}^{*}$, respectively, and $\widetilde{\boldsymbol{\theta}}_{f_{x}}=\boldsymbol{\theta}_{f_{x}}^{*}-\widehat{\boldsymbol{\theta}}_{f_{x}}$ and $\widetilde{\boldsymbol{\theta}}_{g_{x}}=\boldsymbol{\theta}_{g_{x}}^{*}-\widehat{\boldsymbol{\theta}}_{g_{x}}$ are estimation error of $\boldsymbol{\theta}_{f_{x}}^{*}$ and $\boldsymbol{\theta}_{g_{x}}^{*}$, respectively.

Lemma 4. According to

$$
\begin{aligned}
\widetilde{\boldsymbol{\theta}}^{T} \widehat{\boldsymbol{\theta}} & =\frac{1}{2}\left[\widetilde{\boldsymbol{\theta}}^{T}\left(\boldsymbol{\theta}^{*}-\widetilde{\boldsymbol{\theta}}\right)+\left(\boldsymbol{\theta}^{*}-\widehat{\boldsymbol{\theta}}\right)^{T} \widehat{\boldsymbol{\theta}}\right] \\
& =\frac{1}{2}\left[-\|\widetilde{\boldsymbol{\theta}}\|^{2}-\|\widehat{\boldsymbol{\theta}}\|^{2}+\left\|\boldsymbol{\theta}^{*}\right\|^{2}\right]
\end{aligned}
$$

there exist

$$
\begin{aligned}
& \tilde{\boldsymbol{\theta}}^{T} \widehat{\boldsymbol{\theta}} \leq \frac{1}{2}\left[-\|\widehat{\boldsymbol{\theta}}\|^{2}+\left\|\boldsymbol{\theta}^{*}\right\|^{2}\right], \\
& \tilde{\boldsymbol{\theta}}^{T} \widehat{\boldsymbol{\theta}} \leq \frac{1}{2}\left[-\|\widetilde{\boldsymbol{\theta}}\|^{2}+\left\|\boldsymbol{\theta}^{*}\right\|^{2}\right] .
\end{aligned}
$$

Lemma 5 (see [31]). The adaptive law of $\widehat{\boldsymbol{\theta}}$ is designed as

$$
\dot{\hat{\boldsymbol{\theta}}}=\boldsymbol{\Gamma P r o j}(\widehat{\boldsymbol{\theta}}, \boldsymbol{v}, \beta), \quad \boldsymbol{\Gamma}>0, \quad \beta>0,
$$

where

$$
\begin{aligned}
& \operatorname{Proj}(\widehat{\boldsymbol{\theta}}, \boldsymbol{v}, \beta) \\
& = \begin{cases}\boldsymbol{\nu}-\beta \widehat{\boldsymbol{\theta}}, & \text { if }\|\widehat{\boldsymbol{\theta}}\|<\theta^{M} \text { or }\|\widehat{\boldsymbol{\theta}}\|=\theta^{M}, \widehat{\boldsymbol{\theta}}^{T} \boldsymbol{\nu} \leq 0 \\
\boldsymbol{\nu}-\beta \widehat{\boldsymbol{\theta}}-\frac{\hat{\boldsymbol{\theta}} \widehat{\boldsymbol{\theta}}^{T} \boldsymbol{\nu}}{\|\hat{\boldsymbol{\theta}}\|^{2}}, & \text { if }\|\widehat{\boldsymbol{\theta}}\|=\theta^{M}, \widehat{\boldsymbol{\theta}}^{T} \boldsymbol{\nu}>0 ;\end{cases}
\end{aligned}
$$

then $\|\widehat{\boldsymbol{\theta}}(t)\| \leq \theta^{M} ; \theta^{M}$ is positive constant.

\section{Controller Design}

The control objective pursued in this section is to develop an adaptive neural back-stepping controller for an AHV to provide robust tracking of velocity and altitude commands $V_{\text {ref }}$ and $h_{\text {ref }}$. It is assumed that the rigid body states $V, h$, $\gamma, \alpha$, and $Q$ are available for measurement. It is easy to note that the velocity $V$ is mainly related to $\Phi$ and the altitude $h$ is mainly affected by $\delta_{e}$ since the thrust $T$ affects $V$ and $\delta_{e}$ has a dominant contribution to $h$ change in (1). In what follows, the respective control laws $\Phi$ and $\delta_{e}$ will be designed to make $V \rightarrow V_{\text {ref }}$ and $h \rightarrow h_{\text {ref }}$

Assumption 6. Consider sup $\left|\Delta_{x}\right| \leq \Delta_{x}^{M} ; \Delta_{x}^{M}$ is positive constant. Define $\widetilde{\Delta}_{x}^{M}=\Delta_{x}^{M}-\widehat{\Delta}_{x}^{M}$ which stand for the estimation error of $\Delta_{x}^{M}$.

Lemma 7 (see [32]). If $x \in \mathbf{R}$ and $\varepsilon>0$, then $0 \leq|x|-$ $x \tanh (x / \varepsilon) \leq k \varepsilon, k=0.2785$.

3.1. Controller Design for Velocity Subsystem. Differentiating the velocity track error $\widetilde{V}=V-V_{\text {ref }}$ with respect to time results in

$$
\begin{aligned}
\dot{\widetilde{V}}= & f_{V}+g_{V} \Phi+F_{V}+\Delta_{V}-\dot{V}_{\text {ref }} \\
= & \widehat{f}_{V}+\widehat{g}_{V} \Phi^{0}+\left[\left(f_{V}-\widehat{f}_{V}\right)+\left(g_{V}-\widehat{g}_{V}\right) \Phi\right] \\
& +\widehat{g}_{V}\left(\Phi-\Phi^{0}\right)+F_{V}+\Delta_{V}-\dot{V}_{\text {ref }}
\end{aligned}
$$


with

$$
\begin{aligned}
\left(f_{V}\right. & \left.-\widehat{f}_{V}\right)+\left(g_{V}-\widehat{g}_{V}\right) \Phi \\
= & \left(\boldsymbol{\theta}_{f_{V}}^{*}-\widehat{\boldsymbol{\theta}}_{f_{V}}\right)^{T} \boldsymbol{\xi}_{f_{V}}+\left(\boldsymbol{\theta}_{g_{V}}^{*}-\widehat{\boldsymbol{\theta}}_{g_{V}}\right)^{T} \boldsymbol{\xi}_{g_{V}} \Phi+\varepsilon_{f_{V}}^{*} \\
& +\varepsilon_{g_{V}}^{*} \Phi .
\end{aligned}
$$

To eliminate the error between $\Phi$ and $\Phi^{0}$, a novel auxiliary system is introduced as

$$
\dot{\lambda}_{V}=-\kappa_{V} \lambda_{V}-\frac{\widetilde{V} \hat{g}_{V}\left(\Phi-\Phi^{0}\right)}{\lambda_{V}}
$$

where $\kappa_{V}>0$ is a design parameter.

The adaptive law of weight vector can be designed as

$$
\begin{aligned}
& \dot{\hat{\boldsymbol{\theta}}}_{f_{V}}=\boldsymbol{\Gamma}_{f_{V}}\left(\boldsymbol{\xi}_{f_{V}} \widetilde{V}-\beta_{f_{V}} \widehat{\boldsymbol{\theta}}_{f_{V}}\right), \\
& \dot{\hat{\boldsymbol{\theta}}}_{g_{V}}=\boldsymbol{\Gamma}_{g_{V}} \operatorname{Proj}\left(\widehat{\boldsymbol{\theta}}_{g_{V}}, \boldsymbol{\xi}_{g_{V}} \Phi \widetilde{V}, \beta_{g_{V}}\right),
\end{aligned}
$$

where $\boldsymbol{\Gamma}_{f_{V}}=\boldsymbol{\Gamma}_{f_{V}}^{T}, \boldsymbol{\Gamma}_{g_{V}}=\boldsymbol{\Gamma}_{g_{V}}^{T}$, and $\beta_{f_{V}}$ and $\beta_{g_{V}}$ are design parameters.

The adaptive law of $\Delta_{V}^{M}$ is given as follows:

$$
\dot{\widehat{\Delta}}_{V}^{M}=\sigma_{V}\left(|\widetilde{V}|-\beta_{V} \widehat{\Delta}_{V}^{M}\right),
$$

where $\sigma_{V}$ and $\beta_{V}$ are design parameters.

The desired controller $\Phi^{0}$ is introduced as

$$
\begin{aligned}
\Phi^{0} & =\widehat{g}_{V}^{-1}\left[-k_{V, 1} \widetilde{V}-k_{V, 2} \int_{0}^{t} \widetilde{V} d \tau+k_{V, 3} \lambda_{V}-F_{V}+\dot{V}_{\text {ref }}\right. \\
& \left.-\widehat{f}_{V}-\widehat{\Delta}_{V}^{M} \tanh \left(\frac{\widetilde{V}}{\varepsilon_{V}}\right)\right],
\end{aligned}
$$

where $k_{V, 1}$ and $k_{V, 2}$ are positive constants to be designed.

Plugging (23) into (18) results in

$$
\begin{aligned}
\dot{\vec{V}}= & -k_{V, 1} \widetilde{V}-k_{V, 2} \int_{0}^{t} \widetilde{V} d \tau+k_{V, 3} \lambda_{V}+\widetilde{\boldsymbol{\theta}}_{f_{V}}^{T} \xi_{f_{V}} \\
& +\widetilde{\boldsymbol{\theta}}_{g_{V}}^{T} \xi_{g_{V}} \Phi+\varepsilon_{f_{V}}^{*}+\varepsilon_{g_{V}}^{*} \Phi+\widehat{g}_{V}\left(\Phi-\Phi^{0}\right) \\
& +\left[\Delta_{V}-\widehat{\Delta}_{V}^{M} \tanh \left(\frac{\widetilde{V}}{\varepsilon_{V}}\right)\right] .
\end{aligned}
$$

The Lyapunov function can be designed as

$$
\begin{aligned}
W_{V}= & \frac{1}{2} \widetilde{V}^{2}+\frac{k_{V, 2}}{2}\left(\int_{0}^{t} \widetilde{V} d \tau\right)^{2}+\frac{1}{2} \lambda_{V}^{2}+\frac{1}{2 \sigma_{V}}\left(\widetilde{\Delta}_{V}^{M}\right)^{2} \\
& +\frac{1}{2} \widetilde{\boldsymbol{\theta}}_{f_{V}}^{T} \boldsymbol{\Gamma}_{f_{V}}^{-1} \widetilde{\boldsymbol{\theta}}_{f_{V}}+\frac{1}{2} \widetilde{\boldsymbol{\theta}}_{g_{V}}^{T} \boldsymbol{\Gamma}_{g_{V}}^{-1} \widetilde{\boldsymbol{\theta}}_{g_{V}} .
\end{aligned}
$$

Differentiating $W_{V}$ with respect to time results in

$$
\begin{aligned}
\dot{W}_{V}= & -k_{V, 1} \widetilde{V}^{2}+k_{V, 3} \widetilde{V} \lambda_{V}-\kappa_{V} \lambda_{V}^{2}+\widetilde{V}\left(\varepsilon_{f_{V}}^{*}+\varepsilon_{g_{V}}^{*} \Phi\right) \\
& +\widetilde{V}\left[\Delta_{V}-\widehat{\Delta}_{V}^{M} \tanh \left(\frac{\widetilde{V}}{\varepsilon_{V}}\right)\right]+|\widetilde{V}| \widetilde{\Delta}_{V}^{M} \\
& -\beta_{V} \widetilde{\Delta}_{V}^{M} \widehat{\Delta}_{V}^{M}-\beta_{f_{V}} \widetilde{\boldsymbol{\theta}}_{f_{V}}^{T} \widehat{\boldsymbol{\theta}}_{f_{V}}-\beta_{g_{V}} \widetilde{\boldsymbol{\theta}}_{g_{V}}^{T} \widehat{\boldsymbol{\theta}}_{g_{V}} \\
& -v_{f_{V}} \widetilde{\boldsymbol{\theta}}_{f_{V}}^{T} \widehat{\boldsymbol{\theta}}_{f_{V}} \frac{\widehat{\boldsymbol{\theta}}_{f_{V}}^{T} \boldsymbol{\xi}_{f_{V}} \widetilde{V}}{\left\|\widehat{\boldsymbol{\theta}}_{f_{V}}\right\|^{2}}-v_{g_{V}} \widetilde{\boldsymbol{\theta}}_{g_{V}}^{T} \widehat{\boldsymbol{\theta}}_{g_{V}} \frac{\widehat{\boldsymbol{\theta}}_{g_{V}}^{T} \xi_{g_{V}} \Phi \widetilde{V}}{\left\|\widehat{\boldsymbol{\theta}}_{g_{V}}\right\|^{2}} .
\end{aligned}
$$

When $\left\|\widehat{\boldsymbol{\theta}}_{g_{V}}\right\| \leq \theta_{g_{V}}^{M}$ and $\widehat{\boldsymbol{\theta}}_{g_{V}}^{T} \leq 0, v_{f_{V}}=v_{g_{V}}=0$; when $\left\|\widehat{\boldsymbol{\theta}}_{g_{V}}\right\|=$ $\theta_{g_{V}}^{M}$ and $\widehat{\theta}_{g_{V}}^{T}>0, v_{f_{V}}=v_{g_{V}}=1$.

Invoking Lemma 7, we have

$$
\begin{aligned}
& \widetilde{V}\left[\Delta_{V}-\widehat{\Delta}_{V}^{M} \tanh \left(\frac{\widetilde{V}}{\varepsilon_{V}}\right)\right] \\
& \quad \leq|\widetilde{V}| \widetilde{\Delta}_{V}^{M}+\widehat{\Delta}_{V}^{M}\left[|\widetilde{V}|-\widetilde{V} \tanh \left(\frac{\widetilde{V}}{\varepsilon_{V}}\right)\right] \\
& \quad \leq-|\widetilde{V}| \widetilde{\Delta}_{V}^{M}+k_{V}\left|\widehat{\Delta}_{V}^{M}\right| \varepsilon_{V} .
\end{aligned}
$$

Substituting (27) into (26) results in

$$
\begin{aligned}
\dot{W}_{V} \leq & -\mu_{V, 1}\left(\widetilde{V}-\mu_{V, 2} \lambda_{V}\right)^{2} \\
& -\mu_{V, 3}\left[\widetilde{V}-\mu_{V, 4}\left(\varepsilon_{f_{V}}^{*}+\varepsilon_{g_{V}}^{*} \Phi\right)\right]^{2} \\
& +\mu_{V, 5}\left(\varepsilon_{f_{V}}^{*}+\varepsilon_{g_{V}}^{*} \Phi\right)^{2}+k_{V}\left|\widehat{\Delta}_{V}^{M}\right| \varepsilon_{V}-\beta_{V} \widetilde{\Delta}_{V}^{M} \widehat{\Delta}_{V}^{M} \\
& -\beta_{f_{V}} \widetilde{\boldsymbol{\theta}}_{f_{V}}^{T} \widehat{\boldsymbol{\theta}}_{f_{V}}-\beta_{g_{V}} \widetilde{\boldsymbol{\theta}}_{g_{V}}^{T} \widehat{\boldsymbol{\theta}}_{g_{V}},
\end{aligned}
$$

where

$$
\begin{aligned}
& \mu_{V, 1}=\frac{k_{V, 3}^{2}}{\left(4 \kappa_{V}\right)}, \\
& \mu_{V, 2}=\frac{2 \kappa_{V}}{k_{V, 3}}, \\
& \mu_{V, 3}=k_{V, 1}-\mu_{V, 1}, \\
& \mu_{V, 4}=\frac{1}{\left(2 \mu_{V, 3}\right)}, \\
& \mu_{V, 5}=\mu_{V, 3} \mu_{V, 4}^{2} .
\end{aligned}
$$

According to $2 x y \leq c x^{2}+(1 / c) y^{2}$, we obtain

$$
k_{V}\left|\widehat{\Delta}_{V}^{M}\right| \varepsilon_{V} \leq \frac{\beta_{V}\left(\widehat{\Delta}_{V}^{M}\right)^{2}}{2}+\frac{\left(k_{V} \varepsilon_{V}\right)^{2}}{2 \beta_{V}} .
$$

Invoking Lemma 4, it is deduced as

$$
\beta_{V} \widetilde{\Delta}_{V}^{M} \widehat{\Delta}_{V}^{M}=\frac{\beta_{V}}{2}\left[-\left(\widetilde{\Delta}_{V}^{M}\right)^{2}-\left(\widehat{\Delta}_{V}^{M}\right)^{2}+\left(\Delta_{V}^{M}\right)^{2}\right],
$$




$$
\begin{aligned}
& \beta_{f_{V}} \widetilde{\boldsymbol{\theta}}_{f_{V}}^{T} \widehat{\boldsymbol{\theta}}_{f_{V}} \leq \frac{\beta_{f_{V}}}{2}\left[-\left\|\widetilde{\boldsymbol{\theta}}_{f_{V}}\right\|^{2}+\left\|\boldsymbol{\theta}_{f_{V}}^{*}\right\|^{2}\right], \\
& \beta_{g_{V}} \widetilde{\boldsymbol{\theta}}_{g_{V}}^{T} \widehat{\boldsymbol{\theta}}_{g_{V}} \leq \frac{\beta_{g_{V}}}{2}\left[-\left\|\widetilde{\boldsymbol{\theta}}_{g_{V}}\right\|^{2}+\left\|\boldsymbol{\theta}_{g_{V}}^{*}\right\|^{2}\right] .
\end{aligned}
$$

Combining (30)-(33), we have the expression of

$$
\begin{aligned}
\dot{W}_{V} \leq & -\mu_{V, 1}\left(\widetilde{V}-\mu_{V, 2} \lambda_{V}\right)^{2} \\
& -\mu_{V, 3}\left[\widetilde{V}-\mu_{V, 4}\left(\varepsilon_{f_{V}}^{*}+\varepsilon_{g_{V}}^{*} \Phi\right)\right]^{2}-\frac{\beta_{V}}{2}\left(\widetilde{\Delta}_{V}^{M}\right)^{2} \\
& -\frac{\beta_{f_{V}}}{2}\left\|\widetilde{\boldsymbol{\theta}}_{f_{V}}\right\|^{2}-\frac{\beta_{g_{V}}}{2}\left\|\widetilde{\boldsymbol{\theta}}_{g_{V}}\right\|^{2} \\
& +\mu_{V, 5}\left(\varepsilon_{f_{V}}^{*}+\varepsilon_{g_{V}}^{*} \Phi\right)^{2}+\frac{k_{V}^{2}}{2 \beta_{V}} \varepsilon_{V}^{2}+\frac{\beta_{V}}{2}\left(\Delta_{V}^{M}\right)^{2} \\
& +\frac{\beta_{f_{V}}}{2}\left\|\boldsymbol{\theta}_{f_{V}}^{*}\right\|^{2}+\frac{\beta_{g_{V}}}{2}\left\|\boldsymbol{\theta}_{g_{V}}^{*}\right\|^{2} .
\end{aligned}
$$

3.2. Controller Design for Altitude Subsystem. Define the tracking errors of $h, \gamma, \alpha$, and $Q$, respectively, as

$$
\begin{aligned}
& \widetilde{h}=h-h_{\text {ref }}, \\
& \widetilde{\gamma}=\gamma-\gamma_{c}, \\
& \widetilde{\alpha}=\alpha-\alpha_{c}, \\
& \widetilde{Q}=Q-Q_{c} .
\end{aligned}
$$

Differentiating (35)-(38) and invoking (5)-(8) yield

$$
\begin{aligned}
\dot{\tilde{h}}= & V \gamma+\Delta_{h}-\dot{h}_{\text {ref }} \\
= & V \gamma_{c}^{0}+V \widetilde{\gamma}+V\left(\gamma_{c}-\gamma_{c}^{0}\right)+\Delta_{h}-\dot{h}_{\mathrm{ref}}, \\
\dot{\tilde{\gamma}}= & f_{\gamma}+g_{\gamma} \alpha+F_{\gamma}+\Delta_{\gamma}-\dot{\gamma}_{c} \\
= & \widehat{f}_{\gamma}+\widehat{g}_{\gamma} \alpha_{c}^{0}+\left[\left(f_{\gamma}-\widehat{f}_{\gamma}\right)+\left(g_{\gamma}-\widehat{g}_{\gamma}\right) \alpha\right] \\
& +\widehat{g}_{\gamma}\left(\alpha_{c}-\alpha_{c}^{0}\right)+\widehat{g}_{\gamma} \widetilde{\alpha}+F_{\gamma}+\Delta_{\gamma}-\dot{\gamma}_{c}, \\
\dot{\tilde{\alpha}}= & -\left(f_{\gamma}+g_{\gamma} \alpha\right)+Q+F_{\alpha}+\Delta_{\alpha}-\dot{\alpha}_{c} \\
= & -\left(\widehat{f}_{\gamma}+\widehat{g}_{\gamma} \alpha\right)+Q_{c}^{0}-\left[\left(f_{\gamma}-\widehat{f}_{\gamma}\right)+\left(g_{\gamma}-\widehat{g}_{\gamma}\right) \alpha\right] \\
& +\left(Q_{c}-Q_{c}^{0}\right)+\widetilde{Q}+F_{\alpha}+\Delta_{\alpha}-\dot{\alpha}_{c}, \\
\dot{\widetilde{Q}}= & f_{\mathrm{Q}}+g_{\mathrm{Q}} \delta_{e}+\Delta_{\mathrm{Q}}-\dot{Q}_{c} \\
= & \widehat{f}_{\mathrm{Q}}+\widehat{g}_{\mathrm{Q}} \delta_{e}^{0}+\left[\left(f_{\mathrm{Q}}-\widehat{f}_{\mathrm{Q}}\right)+\left(g_{\mathrm{Q}}-\widehat{g}_{\mathrm{Q}}\right) \delta_{e}\right] \\
& +\widehat{g}_{\mathrm{Q}}\left(\delta_{e}-\delta_{e}^{0}\right)+\Delta_{\mathrm{Q}}-\dot{Q}_{c},
\end{aligned}
$$

where

$$
\begin{aligned}
\left(f_{\gamma}-\widehat{f}_{\gamma}\right)+\left(g_{\gamma}-\widehat{g}_{\gamma}\right) \alpha \\
=\left(\boldsymbol{\theta}_{f_{\gamma}}^{*}-\widehat{\boldsymbol{\theta}}_{f_{\gamma}}\right)^{T} \boldsymbol{\xi}_{f_{\gamma}}+\left(\boldsymbol{\theta}_{g_{\gamma}}^{*}-\widehat{\boldsymbol{\theta}}_{g_{\gamma}}\right)^{T} \boldsymbol{\xi}_{g_{\gamma}} \alpha+\varepsilon_{f_{\gamma}}^{*} \\
\quad+\varepsilon_{g_{\gamma}}^{*} \alpha\left(f_{\mathrm{Q}}-\widehat{f}_{\mathrm{Q}}\right)+\left(g_{\mathrm{Q}}-\widehat{g}_{\mathrm{Q}}\right) \delta_{e} \\
=\left(\boldsymbol{\theta}_{f_{\mathrm{Q}}}^{*}-\widehat{\boldsymbol{\theta}}_{f_{\mathrm{Q}}}\right)^{T} \xi_{f_{\mathrm{Q}}}+\left(\boldsymbol{\theta}_{g_{\mathrm{Q}}}^{*}-\widehat{\boldsymbol{\theta}}_{g_{\mathrm{Q}}}\right)^{T} \xi_{g_{\mathrm{Q}}} \delta_{e}+\boldsymbol{\varepsilon}_{f_{\mathrm{Q}}}^{*} \\
\quad+\varepsilon_{g_{\mathrm{Q}}}^{*} \delta_{e} .
\end{aligned}
$$

The auxiliary systems are introduced as

$$
\begin{aligned}
& \dot{\lambda}_{h}=-\kappa_{h} \lambda_{h}-\frac{\tilde{h} V\left(\gamma_{c}-\gamma_{c}^{0}\right)}{\lambda_{h}}, \\
& \dot{\lambda}_{\gamma}=-\kappa_{\gamma} \lambda_{\gamma}-\frac{\widetilde{\gamma} \widehat{g}_{\gamma}\left(\alpha_{c}-\alpha_{c}^{0}\right)}{\lambda_{\gamma}}, \\
& \dot{\lambda}_{\alpha}=-\kappa_{\alpha} \lambda_{\alpha}-\frac{\widetilde{\alpha}\left(Q_{c}-Q_{c}^{0}\right)}{\lambda_{\alpha}}, \\
& \dot{\lambda}_{\mathrm{Q}}=-\kappa_{\mathrm{Q}} \lambda_{\mathrm{Q}}-\frac{\widetilde{\mathrm{Q}} \hat{g}_{\mathrm{Q}}\left(\delta_{e}-\delta_{e}^{0}\right)}{\lambda_{\mathrm{Q}}},
\end{aligned}
$$

where $\kappa_{x}(x=h, \gamma, \alpha, Q)$ stand for design parameters.

The adaptive laws of weight vector can be designed as

$$
\begin{aligned}
& \dot{\hat{\boldsymbol{\theta}}}_{f_{\gamma}}=\boldsymbol{\Gamma}_{f_{\gamma}}\left[\boldsymbol{\xi}_{f_{\gamma}}(\tilde{\gamma}-\widetilde{\alpha})-\beta_{f_{\gamma}} \widehat{\boldsymbol{\theta}}_{f_{\gamma}}\right], \\
& \dot{\hat{\boldsymbol{\theta}}}_{g_{\gamma}}=\boldsymbol{\Gamma}_{g_{\gamma}} \operatorname{Proj}\left[\widehat{\boldsymbol{\theta}}_{g_{\gamma}}, \boldsymbol{\xi}_{g_{\gamma}} \alpha(\tilde{\gamma}-\widetilde{\alpha}), \beta_{g_{\gamma}}\right], \\
& \dot{\hat{\boldsymbol{\theta}}}_{f_{\mathrm{Q}}}=\boldsymbol{\Gamma}_{f_{\mathrm{Q}}}\left(\boldsymbol{\xi}_{f_{\mathrm{Q}}} \widetilde{\boldsymbol{Q}}-\beta_{f_{\mathrm{Q}}} \widehat{\boldsymbol{\theta}}_{f_{\mathrm{Q}}}\right), \\
& \dot{\hat{\boldsymbol{\theta}}}_{g_{\mathrm{Q}}}=\boldsymbol{\Gamma}_{g_{\mathrm{Q}}} \operatorname{Proj}\left[\widehat{\boldsymbol{\theta}}_{g_{\mathrm{Q}}}, \boldsymbol{\xi}_{g_{\mathrm{Q}}} \delta_{e} \widetilde{Q}, \beta_{g_{\mathrm{Q}}}\right],
\end{aligned}
$$

where $\boldsymbol{\Gamma}_{f_{x}}=\boldsymbol{\Gamma}_{f_{x}}^{T}$ and $\boldsymbol{\Gamma}_{g_{x}}=\boldsymbol{\Gamma}_{g_{x}}^{T} ; \beta_{f_{x}}$ and $\beta_{g_{x}}(x=\gamma, Q)$ are design parameters.

The adaptive laws of $\Delta_{x}^{M}(x=h, \gamma, \alpha, Q)$ are given as follows:

$$
\begin{aligned}
& \dot{\hat{\vec{\Delta}}}_{h}^{M}=\sigma_{h}\left(|\widetilde{h}|-\beta_{h} \widehat{\Delta}_{h}^{M}\right) \\
& \dot{\hat{\Delta}}_{\gamma}^{M}=\sigma_{\gamma}\left(|\widetilde{\gamma}|-\beta_{\gamma} \widehat{\Delta}_{\gamma}^{M}\right) \\
& \dot{\widehat{\Delta}}_{\alpha}^{M}=\sigma_{\alpha}\left(|\widetilde{\alpha}|-\beta_{\alpha} \widehat{\Delta}_{\alpha}^{M}\right) \\
& \dot{\widehat{\Delta}}_{\mathrm{Q}}^{M}=\sigma_{\mathrm{Q}}\left(|\widetilde{\mathrm{Q}}|-\beta_{\mathrm{Q}} \widehat{\Delta}_{\mathrm{Q}}^{M}\right),
\end{aligned}
$$

where $\sigma_{x}$ and $\beta_{x}(x=h, \gamma, \alpha, Q)$ are design parameters. 

as

The desired control laws $\gamma_{c}^{0}, \alpha_{c}^{0}, Q_{c}^{0}$, and $\delta_{e}^{0}$ are designed

$$
\begin{aligned}
& \gamma_{c}^{0}=V^{-1}\left[-k_{h, 1} \tilde{h}-k_{h, 2} \int_{0}^{t} \tilde{h} d \tau+k_{h, 3} \lambda_{h}+\dot{h}_{\text {ref }}\right. \\
& \left.-\widehat{\Delta}_{h}^{M} \tanh \left(\frac{\tilde{h}}{\varepsilon_{h}}\right)\right] \\
& \alpha_{c}^{0}=\widehat{g}_{\gamma}^{-1}\left[-k_{\gamma, 1} \tilde{\gamma}-k_{\gamma, 2} \int_{0}^{t} \tilde{\gamma} d \tau+k_{\gamma, 3} \lambda_{\gamma}-\widehat{f}_{\gamma}-F_{\gamma}\right. \\
& \left.-V \widetilde{h}+\dot{\gamma}_{c}-\widehat{\Delta}_{\gamma}^{M} \cdot \tanh \left(\frac{\tilde{\gamma}}{\varepsilon_{\gamma}}\right)-\frac{c_{\gamma} \tilde{\gamma} \alpha^{2}}{2}\right] \text {, } \\
& Q_{c}^{0}=-k_{\alpha, 1} \tilde{\alpha}-k_{\alpha, 2} \int_{0}^{t} \tilde{\alpha} d \tau+k_{\alpha, 3} \lambda_{\alpha}+\widehat{f}_{\gamma}+\widehat{g}_{\gamma} \alpha-F_{\alpha} \\
& -\widehat{g}_{\gamma} \tilde{\gamma}+\dot{\alpha}_{c}-\widehat{\Delta}_{\alpha}^{M} \tanh \left(\frac{\tilde{\alpha}}{\varepsilon_{\alpha}}\right)-\frac{\left(c_{\alpha, 1}+c_{\alpha, 2} \alpha^{2}\right) \tilde{\alpha}}{2}, \\
& \delta_{e}^{0}=\widehat{g}_{\mathrm{Q}}^{-1}\left[-k_{\mathrm{Q}, 1} \widetilde{\mathrm{Q}}-k_{\mathrm{Q}, 2} \int_{0}^{t} \widetilde{\mathrm{Q}} d \tau+k_{\mathrm{Q}, 3} \lambda_{\mathrm{Q}}-\widehat{f}_{\mathrm{Q}}-\widetilde{\alpha}\right. \\
& \left.+\dot{Q}_{c}-\widehat{\Delta}_{\mathrm{Q}} \tanh \left(\frac{\widetilde{\mathrm{Q}}}{\varepsilon_{\mathrm{Q}}}\right)\right]
\end{aligned}
$$

where $k_{x, 1}, k_{x, 2}(x=h, \gamma, \alpha, Q)$ are positive constants to be designed.

Substituting (47)-(50) into (39)-(42) gives

$$
\begin{aligned}
& \dot{\widetilde{h}}=-k_{h, 1} \tilde{h}-k_{h, 2} \int_{0}^{t} \tilde{h} d \tau+k_{h, 3} \lambda_{h}+V \tilde{\gamma}+V\left(\gamma_{c}-\gamma_{c}^{0}\right) \\
& +\left[\Delta_{h}-\widehat{\Delta}_{h}^{M} \tanh \left(\frac{\tilde{h}}{\varepsilon_{h}}\right)\right] \\
& \dot{\tilde{\gamma}}=-k_{\gamma, 1} \tilde{\gamma}-k_{\gamma, 2} \int_{0}^{t} \tilde{\gamma} d \tau+k_{\gamma, 3} \lambda_{\gamma}-V \tilde{h}+\widehat{g}_{\gamma} \widetilde{\alpha} \\
& -\frac{c_{\gamma} \tilde{\gamma} \alpha^{2}}{2}+\widetilde{\boldsymbol{\theta}}_{f_{\gamma}}^{T} \boldsymbol{\xi}_{f_{\gamma}}+\widetilde{\boldsymbol{\theta}}_{g_{\gamma}}^{T} \boldsymbol{\xi}_{g_{\gamma}} \alpha+\varepsilon_{f_{\gamma}}^{*}+\varepsilon_{g_{\gamma}}^{*} \alpha \\
& +\widehat{g}_{\gamma}\left(\alpha_{c}-\alpha_{c}^{0}\right)+\left[\Delta_{\gamma}-\widehat{\Delta}_{\gamma}^{M} \tanh \left(\frac{\tilde{\gamma}}{\varepsilon_{\gamma}}\right)\right] \text {, } \\
& \dot{\tilde{\alpha}}=-k_{\alpha, 1} \tilde{\alpha}-k_{\alpha, 2} \int_{0}^{t} \tilde{\alpha} d \tau+k_{\alpha, 3} \lambda_{\alpha}-\widehat{g}_{\gamma} \tilde{\gamma} \\
& +\frac{\left(c_{\alpha, 1}+c_{\alpha, 2} \alpha^{2}\right) \tilde{\alpha}}{2}+\widetilde{Q}-\left(\widetilde{\boldsymbol{\theta}}_{f_{\gamma}}^{T} \boldsymbol{\xi}_{f_{\gamma}}+\widetilde{\boldsymbol{\theta}}_{g_{\gamma}}^{T} \boldsymbol{\xi}_{g_{\gamma}} \alpha\right) \\
& -\left(\varepsilon_{f_{\gamma}}^{*}+\varepsilon_{g_{\gamma}}^{*} \alpha\right)+\left(Q_{c}-Q_{c}^{0}\right) \\
& +\left[\Delta_{\alpha}-\widehat{\Delta}_{\alpha}^{M} \tanh \left(\frac{\tilde{\alpha}}{\varepsilon_{\alpha}}\right)\right],
\end{aligned}
$$

$$
\begin{aligned}
\dot{\widetilde{Q}}= & -k_{\mathrm{Q}, 1} \widetilde{\mathrm{Q}}-k_{\mathrm{Q}, 2} \int_{0}^{t} \widetilde{\mathrm{Q}} d \tau+k_{\mathrm{Q}, 3} \lambda_{\mathrm{Q}}-\widetilde{\alpha}+\widetilde{\boldsymbol{\theta}}_{f_{\mathrm{Q}}}^{T} \boldsymbol{\xi}_{f_{\mathrm{Q}}} \\
& +\widetilde{\boldsymbol{\theta}}_{g_{\mathrm{Q}}}^{T} \xi_{g_{\mathrm{Q}}} \delta_{e}+\varepsilon_{f_{\mathrm{Q}}}^{*}+\varepsilon_{g_{\mathrm{Q}}}^{*} \delta_{e}+\widehat{g}_{\mathrm{Q}}\left(\delta_{e}-\delta_{e}^{0}\right) \\
& +\left[\Delta_{\mathrm{Q}}-\widehat{\Delta}_{\mathrm{Q}}^{M} \tanh \left(\frac{\widetilde{\mathrm{Q}}}{\varepsilon_{\mathrm{Q}}}\right)\right] .
\end{aligned}
$$

The Lyapunov function can be designed as

$$
\begin{aligned}
W_{h}= & \frac{1}{2} \widetilde{h}^{2}+\frac{k_{h, 2}}{2}\left(\int_{0}^{t} \tilde{h} d \tau\right)^{2}+\frac{1}{2} \lambda_{h}^{2}+\frac{1}{2 \sigma_{h}}\left(\widetilde{\Delta}_{h}^{M}\right)^{2}, \\
W_{\gamma}= & \frac{1}{2} \widetilde{\gamma}^{2}+\frac{k_{\gamma, 2}}{2}\left(\int_{0}^{t} \tilde{\gamma} d \tau\right)^{2}+\frac{1}{2} \lambda_{\gamma}^{2}+\frac{1}{2 \sigma_{\gamma}}\left(\widetilde{\Delta}_{\gamma}^{M}\right)^{2} \\
& +\frac{1}{2} \widetilde{\boldsymbol{\theta}}_{f_{\gamma}}^{T} \boldsymbol{\Gamma}_{f_{\gamma}}^{-1} \widetilde{\boldsymbol{\theta}}_{f_{\gamma}}+\frac{1}{2} \widetilde{\boldsymbol{\theta}}_{g_{\gamma}}^{T} \boldsymbol{\Gamma}_{g_{\gamma}}^{-1} \widetilde{\boldsymbol{\theta}}_{g_{\gamma}}, \\
W_{\alpha}= & \frac{1}{2} \widetilde{\alpha}^{2}+\frac{k_{\alpha, 2}}{2}\left(\int_{0}^{t} \widetilde{\alpha} d \tau\right)^{2}+\frac{1}{2} \lambda_{\alpha}^{2}+\frac{1}{2 \sigma_{\alpha}}\left(\widetilde{\Delta}_{\alpha}^{M}\right)^{2}, \\
W_{\mathrm{Q}}= & \frac{1}{2} \widetilde{Q}^{2}+\frac{k_{\mathrm{Q}, 2}}{2}\left(\int_{0}^{t} \widetilde{\mathrm{Q}} d \tau\right)^{2}+\frac{1}{2} \lambda_{\mathrm{Q}}^{2}+\frac{1}{2 \sigma_{\mathrm{Q}}}\left(\widetilde{\Delta}_{\mathrm{Q}}^{M}\right)^{2} \\
& +\frac{1}{2} \widetilde{\boldsymbol{\theta}}_{f_{\mathrm{Q}}}^{T} \boldsymbol{\Gamma}_{f_{\mathrm{Q}}}^{-1} \widetilde{\boldsymbol{\theta}}_{f_{\mathrm{Q}}}+\frac{1}{2} \widetilde{\boldsymbol{\theta}}_{g_{\mathrm{Q}}}^{T} \boldsymbol{\Gamma}_{g_{\mathrm{Q}}}^{-1} \widetilde{\boldsymbol{\theta}}_{g_{\mathrm{Q}}} .
\end{aligned}
$$
(52) yield

$$
\begin{aligned}
& \dot{W}_{h}=-k_{h, 1} \widetilde{h}^{2}+k_{h, 3} \tilde{h} \lambda_{h}-\kappa_{h} \lambda_{h}^{2}+V \widetilde{h} \tilde{\gamma} \\
& +\tilde{h}\left[\Delta_{h}-\widehat{\Delta}_{h}^{M} \tanh \left(\frac{\widetilde{h}}{\varepsilon_{h}}\right)\right]-\frac{\widetilde{\Delta}_{h}^{M} \dot{\vec{\Delta}}_{h}^{M}}{\sigma_{h}}, \\
& \dot{W}_{\gamma}=-k_{\gamma, 1} \tilde{\gamma}^{2}+k_{\gamma, 3} \tilde{\gamma} \lambda_{\gamma}-\kappa_{\gamma} \lambda_{\gamma}^{2}-V \tilde{\gamma} \tilde{h}+\widehat{g}_{\gamma} \tilde{\gamma} \widetilde{\alpha} \\
& -\frac{c_{\gamma} \tilde{\gamma}^{2} \alpha^{2}}{2}+\widetilde{\gamma}\left(\widetilde{\boldsymbol{\theta}}_{f_{\gamma}}^{T} \boldsymbol{\xi}_{f_{\gamma}}+\widetilde{\boldsymbol{\theta}}_{g_{\gamma}}^{T} \boldsymbol{\xi}_{g_{\gamma}} \alpha\right) \\
& +\widetilde{\gamma}\left(\varepsilon_{f_{\gamma}}^{*}+\varepsilon_{g_{\gamma}}^{*} \alpha\right) \\
& +\widetilde{\gamma}\left[\Delta_{\gamma}-\widehat{\Delta}_{\gamma}^{M} \tanh \left(\frac{\tilde{\gamma}}{\varepsilon_{\gamma}}\right)\right]-\frac{\widetilde{\Delta}_{\gamma}^{M} \dot{\widehat{\Delta}}_{\gamma}^{M}}{\sigma_{\gamma}} \\
& +\widetilde{\boldsymbol{\theta}}_{f_{\gamma}}^{T} \boldsymbol{\Gamma}_{f_{\gamma}}^{-1} \dot{\hat{\boldsymbol{\theta}}}_{f_{\gamma}}+\widetilde{\boldsymbol{\theta}}_{g_{\gamma}}^{T} \boldsymbol{\Gamma}_{g_{\gamma}}^{-1} \dot{\hat{\boldsymbol{\theta}}}_{g_{\gamma}}, \\
& \dot{W}_{\alpha}=-k_{\alpha, 1} \widetilde{\alpha}^{2}+k_{\alpha, 3} \widetilde{\alpha} \lambda_{\alpha}-\kappa_{\alpha} \lambda_{\alpha}^{2}-\widehat{g}_{\gamma} \tilde{\alpha} \widetilde{\gamma}+\widetilde{\alpha} \widetilde{Q} \\
& -\frac{\left(c_{\alpha, 1}+c_{\alpha, 2} \alpha^{2}\right) \tilde{\alpha}^{2}}{2}-\widetilde{\alpha}\left(\widetilde{\boldsymbol{\theta}}_{f_{\gamma}}^{T} \boldsymbol{\xi}_{f_{\gamma}}+\widetilde{\boldsymbol{\theta}}_{g_{\gamma}}^{T} \boldsymbol{\xi}_{g_{\gamma}} \alpha\right) \\
& -\tilde{\alpha}\left(\varepsilon_{f_{\gamma}}^{*}+\varepsilon_{g_{\gamma}}^{*} \alpha\right) \\
& +\widetilde{\alpha}\left[\Delta_{\alpha}-\widehat{\Delta}_{\alpha}^{M} \tanh \left(\frac{\widetilde{\alpha}}{\varepsilon_{\alpha}}\right)\right]-\frac{\widetilde{\Delta}_{\alpha}^{M} \dot{\widehat{\Delta}}_{\alpha}^{M}}{\sigma_{\alpha}},
\end{aligned}
$$




$$
\begin{aligned}
\dot{W}_{\mathrm{Q}}= & -k_{\mathrm{Q}, 1} \widetilde{\mathrm{Q}}^{2}+k_{\mathrm{Q}, 3} \widetilde{\mathrm{Q}} \lambda_{\mathrm{Q}}-\kappa_{\mathrm{Q}} \lambda_{\mathrm{Q}}^{2}-\widetilde{\mathrm{Q}} \widetilde{\alpha} \\
& +\widetilde{\mathrm{Q}}\left(\widetilde{\boldsymbol{\theta}}_{f_{\mathrm{Q}}}^{T} \boldsymbol{\xi}_{f_{\mathrm{Q}}}+\widetilde{\boldsymbol{\theta}}_{g_{\mathrm{Q}}}^{T} \boldsymbol{\xi}_{g_{\mathrm{Q}}} \delta_{e}\right) \\
& +\widetilde{\mathrm{Q}}\left(\varepsilon_{f_{\mathrm{Q}}}^{*}+\varepsilon_{g_{\mathrm{Q}}}^{*} \delta_{e}\right) \\
& +\widetilde{\mathrm{Q}}\left[\Delta_{\mathrm{Q}}-\widehat{\Delta}_{\mathrm{Q}}^{M} \tanh \left(\frac{\widetilde{\mathrm{Q}}}{\varepsilon_{\mathrm{Q}}}\right)\right]-\frac{\widetilde{\Delta}_{\mathrm{Q}}^{M} \dot{\hat{\Delta}}_{\mathrm{Q}}^{M}}{\sigma_{\mathrm{Q}}} \\
& +\widetilde{\boldsymbol{\theta}}_{f_{\mathrm{Q}}}^{T} \boldsymbol{\Gamma}_{f_{\mathrm{Q}}}^{-1} \dot{\boldsymbol{\theta}}_{f_{\mathrm{Q}}}+\widetilde{\boldsymbol{\theta}}_{g_{\mathrm{Q}}}^{T} \boldsymbol{\Gamma}_{g_{\mathrm{Q}}}^{-1} \dot{\hat{\boldsymbol{\theta}}}_{g_{\mathrm{Q}}} \cdot
\end{aligned}
$$

Invoking Lemma 5, we can derive

$$
\begin{aligned}
& \tilde{x}\left[\Delta_{x}-\widehat{\Delta}_{x}^{M} \tanh \left(\frac{\tilde{x}}{\varepsilon_{x}}\right)\right] \\
& \leq|\widetilde{x}| \widetilde{\Delta}_{x}^{M}+\widehat{\Delta}_{x}^{M}\left[|\widetilde{x}|-\tilde{x} \tanh \left(\frac{\tilde{x}}{\varepsilon_{x}}\right)\right] \\
& \leq|\widetilde{x}| \widetilde{\Delta}_{x}^{M}+k_{x}\left|\widehat{\Delta}_{x}^{M}\right| \varepsilon_{x},
\end{aligned}
$$

where $x \in\{h, \gamma, \alpha, Q\}$.

The following inequalities hold:

$$
\begin{aligned}
\widetilde{\gamma}_{g_{\gamma}}^{*} \alpha \leq & \frac{c_{\gamma} \widetilde{\gamma}^{2} \alpha^{2}}{2}+\frac{\varepsilon_{g_{\gamma}}^{* 2}}{2 c_{\gamma}} \\
-\widetilde{\alpha}\left(\varepsilon_{f_{\gamma}}^{*}+\varepsilon_{g_{\gamma}}^{*} \alpha\right)= & -\widetilde{\alpha}_{f_{\gamma}}^{*}-\widetilde{\alpha} \alpha \varepsilon_{g_{\gamma}}^{*} \\
\leq & \frac{c_{\alpha, 1} \widetilde{\alpha}^{2}}{2}+\frac{\varepsilon_{f_{\gamma}}^{* 2}}{2 c_{\alpha, 1}}+\frac{c_{\alpha, 2} \widetilde{\alpha}^{2} \alpha^{2}}{2}+\frac{\varepsilon_{g_{\gamma}}^{* 2}}{2 c_{\alpha, 2}} \\
= & \widetilde{\alpha}^{2}\left(\frac{c_{\alpha, 1}}{2}+\frac{c_{\alpha, 2} \alpha^{2}}{2}\right)+\frac{\varepsilon_{f_{\gamma}}^{* 2}}{2 c_{\alpha, 1}} \\
& +\frac{\varepsilon_{g_{\gamma}}^{* 2}}{2 c_{\alpha, 2}},
\end{aligned}
$$

where $c_{\gamma}>0, c_{\alpha, 1}>0$, and $c_{\alpha, 2}>0$.

Plugging (44)-(46) and (57)-(58) into (56) results in

$$
\begin{aligned}
\dot{W}_{h}= & -\mu_{h, 1}\left(\widetilde{h}-\mu_{h, 2} \lambda_{h}\right)^{2}-\mu_{h, 3} \widetilde{h}^{2}+V \widetilde{h} \widetilde{\gamma} \\
& +k_{h}\left|\widehat{\Delta}_{h}^{M}\right| \varepsilon_{h}-\beta_{h} \widetilde{\Delta}_{h}^{M} \widehat{\Delta}_{h}^{M}, \\
\dot{W}_{\gamma}+\dot{W}_{\alpha} \leq & -\mu_{\gamma, 1}\left(\tilde{\gamma}-\mu_{\gamma, 2} \lambda_{\gamma}\right)^{2}-\mu_{\alpha, 1}\left(\widetilde{\alpha}-\mu_{\alpha, 2} \lambda_{\alpha}\right)^{2} \\
& -\mu_{\gamma, 3}\left(\tilde{\gamma}-\mu_{\gamma, 4} \varepsilon_{f_{\gamma}}^{*}\right)^{2}-\mu_{\alpha, 3} \widetilde{\alpha}^{2} \\
& +\left(\mu_{\gamma, 5}+\frac{1}{2 c_{\alpha, 1}}\right) \varepsilon_{f_{\gamma}}^{* 2} \\
& +\left(\frac{1}{2 c_{\gamma}}+\frac{1}{2 c_{\alpha, 2}}\right) \varepsilon_{g_{\gamma}}^{* 2}-V \widetilde{\gamma} \widetilde{h}+\widetilde{\alpha} \widetilde{Q} \\
& +k_{\gamma}\left|\widehat{\Delta}_{\gamma}^{M}\right| \varepsilon_{\gamma}+k_{\alpha}\left|\widehat{\Delta}_{\alpha}^{M}\right| \varepsilon_{\alpha}-\beta_{\gamma} \widetilde{\Delta}_{\gamma}^{M} \widehat{\Delta}_{\gamma}^{M}
\end{aligned}
$$

$$
\begin{aligned}
& -\beta_{\alpha} \widetilde{\Delta}_{\alpha}^{M} \widehat{\Delta}_{\alpha}^{M}-\beta_{f_{\gamma}} \widetilde{\boldsymbol{\theta}}_{f_{\gamma}}^{T} \widehat{\boldsymbol{\theta}}_{f_{\gamma}}-\beta_{g_{\gamma}} \widetilde{\boldsymbol{\theta}}_{g_{\gamma}}^{T} \widehat{\boldsymbol{\theta}}_{g_{\gamma}} \\
& -v_{f_{\gamma}} \widetilde{\boldsymbol{\theta}}_{f_{\gamma}}^{T} \widehat{\boldsymbol{\theta}}_{f_{\gamma}} \frac{\widehat{\boldsymbol{\theta}}_{f_{\gamma}}^{T} \boldsymbol{\xi}_{f_{\gamma}}(\tilde{\gamma}-\tilde{\alpha})}{\left\|\hat{\boldsymbol{\theta}}_{f_{\gamma}}\right\|^{2}} \\
& -v_{g_{\gamma}} \widetilde{\boldsymbol{\theta}}_{g_{\gamma}}^{T} \widehat{\boldsymbol{\theta}}_{g_{\gamma}} \frac{\widehat{\boldsymbol{\theta}}_{g_{\gamma}}^{T} \boldsymbol{\xi}_{g_{\gamma}} \alpha(\widetilde{\gamma}-\widetilde{\alpha})}{\left\|\widehat{\boldsymbol{\theta}}_{g_{\gamma}}\right\|^{2}},
\end{aligned}
$$$$
\dot{W}_{\mathrm{Q}} \leq-\mu_{\mathrm{Q}, 1}\left(\widetilde{\mathrm{Q}}-\mu_{\mathrm{Q}, 2} \lambda_{\mathrm{Q}}\right)^{2}
$$$$
-\mu_{\mathrm{Q}, 3}\left[\widetilde{\mathrm{Q}}-\mu_{\mathrm{Q}, 4}\left(\varepsilon_{f_{\mathrm{Q}}}^{*}+\varepsilon_{g_{\mathrm{Q}}}^{*} \delta_{e}\right)\right]^{2}
$$$$
+\mu_{\mathrm{Q}, 5}\left(\varepsilon_{f_{\mathrm{Q}}}^{*}+\varepsilon_{g_{\mathrm{Q}}}^{*} \delta_{e}\right)^{2}-\widetilde{\mathrm{Q}} \widetilde{\alpha}
$$$$
+\widetilde{Q}\left(\Delta_{Q}-\widehat{\Delta}_{Q}\right)-\beta_{f_{Q}} \widetilde{\boldsymbol{\theta}}_{f_{Q}}^{T} \widehat{\boldsymbol{\theta}}_{f_{\mathrm{Q}}}
$$$$
-\beta_{g_{\mathrm{Q}}} \widetilde{\boldsymbol{\theta}}_{g_{\mathrm{Q}}}^{T} \widehat{\boldsymbol{\theta}}_{g_{\mathrm{Q}}}-v_{f_{\mathrm{Q}}} \tilde{\boldsymbol{\theta}}_{f_{\mathrm{Q}}}^{T} \widehat{\boldsymbol{\theta}}_{f_{\mathrm{Q}}} \frac{\widehat{\boldsymbol{\theta}}_{f_{\mathrm{Q}}}^{T} \boldsymbol{\xi}_{f_{\mathrm{Q}}} \widetilde{\mathrm{Q}}}{\left\|\widehat{\boldsymbol{\theta}}_{f_{\mathrm{Q}}}\right\|^{2}}
$$$$
-v_{g_{\mathrm{Q}}} \widetilde{\boldsymbol{\theta}}_{g_{\mathrm{Q}}}^{T} \widehat{\boldsymbol{\theta}}_{g_{\mathrm{Q}}} \frac{\widehat{\boldsymbol{\theta}}_{g_{\mathrm{Q}}}^{T} \boldsymbol{\xi}_{g_{\mathrm{Q}}} \delta_{e} \widetilde{Q}}{\left\|\widehat{\boldsymbol{\theta}}_{g_{\mathrm{Q}}}\right\|^{2}},
$$

where $\mu_{x, 1}=k_{x, 3}^{2} /\left(4 \kappa_{x}\right), \mu_{x, 2}=2 \kappa_{x} / k_{x, 3}, \mu_{x, 3}=k_{x, 1}-\mu_{x, 1}$, $\mu_{x, 4}=1 /\left(2 \mu_{x, 3}\right)$, and $\mu_{x, 5}=\mu_{x, 3} \mu_{x, 4}^{2}, x \in\{h, \gamma, \alpha, Q\}$.

When $\left\|\widehat{\boldsymbol{\theta}}_{g_{x}}\right\| \leq \theta_{g_{x}}^{M}$ and $\widehat{\boldsymbol{\theta}}_{g_{x}}^{T} \leq 0, v_{f_{x}}=v_{g_{x}}=0(x=$ $h, \gamma, \alpha, Q)$; when $\left\|\widehat{\boldsymbol{\theta}}_{g_{x}}\right\|=\theta_{g_{x}}^{M}$ and $\widehat{\boldsymbol{\theta}}_{g_{x}}^{T}>0, v_{f_{x}}=v_{g_{x}}=1(x=$ $h, \gamma, \alpha, Q)$.

Invoking Lemma 4, we can derive

$$
\begin{aligned}
& k_{x}\left|\widehat{\Delta}_{x}^{M}\right| \varepsilon_{x} \leq \frac{\beta_{x}\left(\widehat{\Delta}_{x}^{M}\right)^{2}}{2}+\frac{\left(k_{x} \varepsilon_{x}\right)^{2}}{2 \beta_{x}}, \\
& \beta_{x} \widetilde{\Delta}_{x}^{M} \widehat{\Delta}_{x}^{M}=\frac{\beta_{x}}{2}\left[-\left(\widetilde{\Delta}_{x}^{M}\right)^{2}-\left(\widehat{\Delta}_{x}^{M}\right)^{2}+\left(\Delta_{x}^{M}\right)^{2}\right], \\
& \beta_{f_{x}} \widetilde{\boldsymbol{\theta}}_{f_{x}}^{T} \widehat{\boldsymbol{\theta}}_{f_{x}} \leq \frac{\beta_{f_{x}}}{2}\left[-\left\|\widetilde{\boldsymbol{\theta}}_{f_{x}}\right\|^{2}+\left\|\boldsymbol{\theta}_{f_{x}}^{*}\right\|^{2}\right], \\
& \beta_{g_{x}} \widetilde{\boldsymbol{\theta}}_{g_{x}}^{T} \widehat{\boldsymbol{\theta}}_{g_{x}} \leq \frac{\beta_{g_{x}}}{2}\left[-\left\|\widetilde{\boldsymbol{\theta}}_{g_{x}}\right\|^{2}+\left\|\boldsymbol{\theta}_{g_{x}}^{*}\right\|^{2}\right] .
\end{aligned}
$$

Combining (60)-(63), we have the expression of

$$
\begin{aligned}
\dot{W}_{h}= & -\mu_{h, 1}\left(\widetilde{h}-\mu_{h, 2} \lambda_{h}\right)^{2}-\mu_{h, 3} \widetilde{h}^{2}-\frac{\beta_{V}}{2}\left(\widetilde{\Delta}_{h}^{M}\right)^{2} \\
& +V \widetilde{h} \widetilde{\gamma}+\frac{k_{h}^{2}}{2 \beta_{h}} \varepsilon_{h}^{2}+\frac{\beta_{h}}{2}\left(\Delta_{h}^{M}\right)^{2},
\end{aligned}
$$




$$
\begin{array}{rlrl}
\dot{W}_{\gamma}+\dot{W}_{\alpha} \leq & -\mu_{\gamma, 1}\left(\tilde{\gamma}-\mu_{\gamma, 2} \lambda_{\gamma}\right)^{2}-\mu_{\alpha, 1}\left(\widetilde{\alpha}-\mu_{\alpha, 2} \lambda_{\alpha}\right)^{2} & +\mu_{V, 5}\left(\varepsilon_{f_{V}}^{*}+\varepsilon_{g_{V}}^{*} \Phi\right)^{2}+\left(\mu_{\gamma, 5}+\frac{1}{2 a_{\alpha, 1}}\right) \varepsilon_{f_{\gamma}}^{* 2} \\
& -\mu_{\gamma, 3}\left(\widetilde{\gamma}-\mu_{\gamma, 4} \varepsilon_{f_{\gamma}}^{*}\right)^{2}-\mu_{\alpha, 3} \widetilde{\alpha}^{2} & +\left(\frac{1}{2 c_{\gamma}}+\frac{1}{2 c_{\alpha, 2}}\right) \cdot \varepsilon_{g_{\gamma}}^{* 2}+\mu_{\mathrm{Q}, 5}\left(\varepsilon_{f_{\mathrm{Q}}}^{*}+\varepsilon_{g_{Q}}^{*} \delta_{e}\right)^{2} \\
& -\frac{\beta_{\gamma}}{2}\left(\widetilde{\Delta}_{\gamma}^{M}\right)^{2}-\frac{\beta_{\alpha}}{2}\left(\widetilde{\Delta}_{\alpha}^{M}\right)^{2} & +\frac{1}{2} \sum_{x} \beta_{f_{x}}\left\|\boldsymbol{\theta}_{f_{x}}^{*}\right\|^{2}+\frac{1}{2} \sum_{x} \beta_{g_{x}}\left\|\boldsymbol{\theta}_{g_{x}}^{*}\right\|^{2} \\
& -\frac{\beta_{f_{\gamma}}}{2}\left\|\widetilde{\boldsymbol{\theta}}_{f_{\gamma}}\right\|^{2}-\frac{\beta_{g_{\gamma}}}{2}\left\|\widetilde{\boldsymbol{\theta}}_{g_{\gamma}}\right\|^{2}-V \widetilde{\gamma} \widetilde{h} & +\frac{1}{2} \sum_{x} \frac{\left(k_{x} \varepsilon_{x}\right)^{2}}{\beta_{x}}+\frac{1}{2} \sum_{x} \beta_{x}\left(\Delta_{x}^{M}\right)^{2}, \\
& +\widetilde{\alpha} \widetilde{Q}+\left(\mu_{\gamma, 5}+\frac{1}{2 c_{\alpha, 1}}\right) \varepsilon_{f_{\gamma}}^{* 2} & (65) &
\end{array}
$$$$
+\left(\frac{1}{2 c_{\gamma}}+\frac{1}{2 c_{\alpha, 2}}\right) \varepsilon_{g_{\gamma}}^{* 2}+\frac{k_{\gamma}^{2}}{2 \beta_{\gamma}} \varepsilon_{\gamma}^{2}
$$$$
+\frac{k_{\alpha}^{2}}{2 \beta_{\alpha}} \varepsilon_{\alpha}^{2}+\frac{\beta_{\gamma}}{2}\left(\Delta_{\gamma}^{M}\right)^{2}+\frac{\beta_{\alpha}}{2}\left(\Delta_{\alpha}^{M}\right)^{2}
$$$$
+\frac{\beta_{f_{\gamma}}}{2}\left\|\boldsymbol{\theta}_{f_{\gamma}}^{*}\right\|^{2}+\frac{\beta_{g_{\gamma}}}{2}\left\|\boldsymbol{\theta}_{g_{\gamma}}^{*}\right\|^{2}
$$

where $x \in\{V, h, \gamma, \alpha, Q\}$.

Let

$$
\begin{aligned}
\Sigma & =\mu_{V, 5}\left(\varepsilon_{f_{V}}^{M}+\varepsilon_{g_{V}}^{M} \Phi^{M}\right)^{2}+\left(\mu_{\gamma, 5}+\frac{1}{2 c_{\alpha, 1}}\right) \cdot\left(\varepsilon_{f_{\gamma}}^{M}\right)^{2} \\
& +\left(\frac{1}{2 c_{\gamma}}+\frac{1}{2 c_{\alpha, 2}}\right)\left(\varepsilon_{g_{\gamma}}^{M}\right)^{2}+\mu_{\mathrm{Q}, 5}\left(\varepsilon_{f_{\mathrm{Q}}}^{M}+\varepsilon_{g_{\mathrm{Q}}}^{M} \delta_{e}^{M}\right)^{2} \\
& +\frac{1}{2} \sum_{x}\left[\frac{\left(k_{x} \varepsilon_{x}\right)^{2}}{\beta_{x}}+\beta_{x}\left(\Delta_{x}^{M}\right)^{2}+\beta_{f_{x}}\left(\boldsymbol{\theta}_{f_{x}}^{M}\right)^{2}\right. \\
& \left.+\beta_{g_{x}}\left(\boldsymbol{\theta}_{g_{x}}^{M}\right)^{2}\right] .
\end{aligned}
$$

3.3. Stability Analysis. The Lyapunov function can be designed as

$$
W=W_{V}+W_{h}+W_{\gamma}+W_{\alpha}+W_{\mathrm{Q}} .
$$

Combining (34) and (60)-(63) and differentiating $W$ with respect to time result in

$$
\begin{aligned}
\dot{W} \leq & -\mu_{V, 3}\left[\widetilde{V}-\mu_{V, 4}\left(\varepsilon_{f_{V}}^{*}+\varepsilon_{g_{V}}^{*} \Phi\right)\right]^{2}-\mu_{h, 3} \widetilde{h}^{2} \\
& -\mu_{\gamma, 3}\left(\tilde{\gamma}-\mu_{\gamma, 4} \varepsilon_{f_{\gamma}}^{*}\right)^{2}-\mu_{\alpha, 3} \widetilde{\alpha}^{2} \\
& -\mu_{\mathrm{Q}, 3}\left[\widetilde{Q}-\mu_{\mathrm{Q}, 4}\left(\varepsilon_{f_{Q}}^{*}+\varepsilon_{g_{Q}}^{*} \delta_{e}\right)\right]^{2} \\
& -\sum_{x} \mu_{x, 1}\left(\tilde{x}-\mu_{x, 2} \lambda_{x}\right)^{2}-\frac{1}{2} \sum_{x} \beta_{x}\left(\widetilde{\Delta}_{x}^{M}\right)^{2} \\
& -\frac{1}{2} \sum_{x} \beta_{f_{x}}\left\|\widetilde{\boldsymbol{\theta}}_{f_{x}}\right\|^{2}-\frac{1}{2} \sum_{x} \beta_{g_{x}}\left\|\widetilde{\boldsymbol{\theta}}_{g_{x}}\right\|^{2}
\end{aligned}
$$

Define

$$
\begin{aligned}
\Omega_{\widetilde{V}} & =\left\{\widetilde{V}|| \widetilde{V} \mid \leq \mu_{V, 4}\left(\varepsilon_{f_{V}}^{M}+\varepsilon_{g_{V}}^{M} \Phi^{M}\right)+\sqrt{\frac{\Sigma}{\mu_{V, 3}}}\right\}, \\
\Omega_{\widetilde{h}} & =\left\{\widetilde{h}|| \widetilde{h} \mid \leq \sqrt{\frac{\Sigma}{\mu_{h, 3}}}\right\}, \\
\Omega_{\tilde{\gamma}} & =\left\{\widetilde{\gamma}|| \widetilde{\gamma} \mid \leq \mu_{\gamma, 4} \varepsilon_{f_{\gamma}}^{M}+\sqrt{\frac{\Sigma}{\mu_{\gamma, 3}}}\right\}, \\
\Omega_{\widetilde{\alpha}} & =\left\{\widetilde{\alpha}|| \widetilde{\alpha} \mid \leq \sqrt{\frac{\Sigma}{\mu_{\alpha, 3}}}\right\}, \\
\Omega_{\widetilde{Q}} & =\left\{\widetilde{Q}|| \widetilde{Q} \mid \leq \mu_{\mathrm{Q}, 4}\left(\varepsilon_{f_{\mathrm{Q}}}^{M}+\varepsilon_{g_{Q}}^{M} \delta_{e}^{M}\right)+\sqrt{\frac{\Sigma}{\mu_{\mathrm{Q}, 3}}}\right\}, \\
\Omega_{\widetilde{\Delta}_{x}^{M}} & =\left\{\widetilde{\Delta}_{x}^{M} \mid\left\|\widetilde{\Delta}_{x}^{M}\right\| \leq \sqrt{\frac{\Sigma}{\beta_{x}}}\right\}, \\
\Omega_{\tilde{\boldsymbol{\theta}}_{f_{x}}} & =\left\{\widetilde{\boldsymbol{\theta}}_{f_{x}} \mid\left\|\widetilde{\boldsymbol{\theta}}_{f_{x}}\right\| \leq \sqrt{\frac{\Sigma}{\beta_{f_{x}}}}\right\}, \\
\Omega_{\tilde{\boldsymbol{\theta}}_{g_{x}}} & =\left\{\widetilde{\boldsymbol{\theta}}_{g_{x}} \mid\left\|\widetilde{\boldsymbol{\theta}}_{g_{x}}\right\| \leq \sqrt{\frac{\Sigma}{\beta_{g_{x}}}}\right\},
\end{aligned}
$$

where $\Phi^{M}=\max \left\{\left|\Phi^{L}\right|,\left|\Phi^{U}\right|\right\} ; \delta_{e}^{L}=\max \left\{\left|\delta_{e}^{L}\right|,\left|\delta_{e}^{U}\right|\right\}$. 
Let

$$
\begin{aligned}
k_{x, 1} & >\frac{k_{x, 3}^{2}}{\left(4 \kappa_{x}\right)}, k_{x, 2}>0, k_{x, 3}>0, \kappa_{x}>0, \\
\Gamma_{f_{x}} & =\Gamma_{f_{x}}^{T}>0, \Gamma_{g_{x}}=\Gamma_{g_{x}}^{T}>0, \beta_{f_{x}}>0, \beta_{g_{x}}>0, \\
\sigma_{x} & >0, \beta_{x}>0 ;
\end{aligned}
$$

If $\widetilde{x}, \widetilde{\boldsymbol{\theta}}_{f_{x}}, \widetilde{\boldsymbol{\theta}}_{g_{x}}$ and $\widetilde{\Delta}_{x}^{M}, \tilde{x} \in\{\widetilde{V}, \widetilde{h}, \widetilde{\gamma}, \widetilde{\alpha}, \widetilde{Q}\}$, do not belong to the compact set $\Omega_{\tilde{x}}, \Omega_{\tilde{\theta}_{f_{x}}}, \Omega_{\tilde{\boldsymbol{\theta}}_{g_{x}}}$ and $\Omega_{\widetilde{\Delta}_{x}^{M}}$, respectively, $\dot{W} \leq 0 . \tilde{x}$, $\tilde{\boldsymbol{\theta}}_{f_{x}}, \widetilde{\boldsymbol{\theta}}_{g_{x}}$, and $\widetilde{\Delta}_{x}^{M}$ are bounded. Further, $\lambda_{x}$ also is bounded according to (66). By regulating the designed parameters, the radius of $\Omega_{\tilde{x}}, \Omega_{\widetilde{W}_{f_{x}}}, \Omega_{\widetilde{W}_{g_{x}}}$, and $\Omega_{\widetilde{\Delta}_{x}^{M}}$ can be made arbitrarily small.

Theorem 8. Consider the system of (1) under Assumptions 3 and 6, auxiliary analytical system (20) and (44), controllers (23) and (47)-(50), adaptive laws (21) and (45), and upper bound estimation of system error (22) and (46). If (71) is satisfied, all the signals involved are bounded.

\section{Simulation Results}

Simulations are done to illustrate the effectiveness of the robust adaptive approximate back-stepping control scheme proposed in the previous section. The vehicle model parameters are referred to in [27]. The damping of flexibility is $\zeta_{i}=0.02, i=1,2,3$, and vibrational frequencies are $\omega_{1}=$ $27.8944 \mathrm{rad} / \mathrm{s}, \omega_{2}=76.8933 \mathrm{rad} / \mathrm{s}$, and $\omega_{3}=150.7526 \mathrm{rad} / \mathrm{s}$.

The aircraft is commanded to perform a cruising flight mission that the initial height is $25908 \mathrm{~m}$ and dynamic pressure is $95760 \mathrm{~Pa}$. The initial trim conditions are designed as follows: $\gamma=0^{\circ}, \alpha=0.6132^{\circ}, Q=0^{\circ} / \mathrm{s}, \Phi=0.1329, \delta_{e}=$ $9.0081^{\circ}, \eta_{1}=1.889 \mathrm{~m} \cdot \mathrm{kg}^{0.5} / \mathrm{m}^{0.5}, \eta_{2}=-0.028 \mathrm{~m} \cdot \mathrm{kg}^{0.5} / \mathrm{m}^{0.5}$, $\eta_{3}=-0.007323 \mathrm{~m} \cdot \mathrm{kg}^{0.5} / \mathrm{m}^{0.5}$, and $\dot{\eta}_{i}=0 \mathrm{~m} / \mathrm{s} \cdot \mathrm{kg}^{0.5} / \mathrm{m}^{0.5}$, $i=1,2,3$.

The reference commands of velocity and altitude are generated via the following filters:

$$
\begin{aligned}
& \frac{V_{\text {ref }}(s)}{V_{c}(s)}=\frac{0.03^{2}}{s^{2}+2 \times 0.95 \times 0.03 \times s+0.03^{2}}, \\
& \frac{h_{\text {ref }}(s)}{h_{c}(s)}=\frac{0.03^{2}}{s^{2}+2 \times 0.95 \times 0.03 \times s+0.03^{2}} .
\end{aligned}
$$

The control laws are limited by $\Phi \in[0.1,1.2], \delta_{e} \in$ $\left[-15^{\circ}, 15^{\circ}\right], \alpha_{c} \in\left[-5^{\circ}, 5^{\circ}\right], \gamma_{c} \in\left[-5^{\circ}, 5^{\circ}\right]$, and $Q_{c} \in$ $\left[-10^{\circ} / \mathrm{s}, 10^{\circ} / \mathrm{s}\right]$. The bandwidth of $\Phi, \delta_{e}, \gamma_{c}, \alpha_{c}$, and $Q_{c}$ is limited by $20 \mathrm{rad} / \mathrm{s}, 20 \mathrm{rad} / \mathrm{s}, 30 \mathrm{rad} / \mathrm{s}, 40 \mathrm{rad} / \mathrm{s}$, and $60 \mathrm{rad} / \mathrm{s}$, respectively.

The design parameters of controller are proposed as follows: $\kappa_{x}=0.1, \varepsilon_{x}=0.01, k_{V, 1}=k_{h, 1}=0.4, k_{\gamma \cdot 1}=$ $k_{\alpha \cdot 1}=k_{\mathrm{Q} \cdot 1}=1, k_{x \cdot 2}=0.01$, and $k_{x \cdot 3}=0.1$. The adaptive law of RBF is considered as follows: $\Gamma_{f_{V}}=0.01 \times I_{625 \times 625}$, $\Gamma_{g_{V}}=0.1 \times I_{625 \times 625}, \Gamma_{f_{\gamma}}=0.01 \times I_{125 \times 125}, \Gamma_{g_{\gamma}}=0.1 \times I_{625 \times 625}$, $\Gamma_{f_{Q}}=0.01 \times I_{625 \times 625}, \Gamma_{g_{Q}}=0.1 \times I_{3125 \times 3125}, \beta_{f_{x}}=\beta_{g_{x}}=0.1$, $\beta_{x}=0.1, \sigma_{x}=1$, and the upper bound of weight vectors
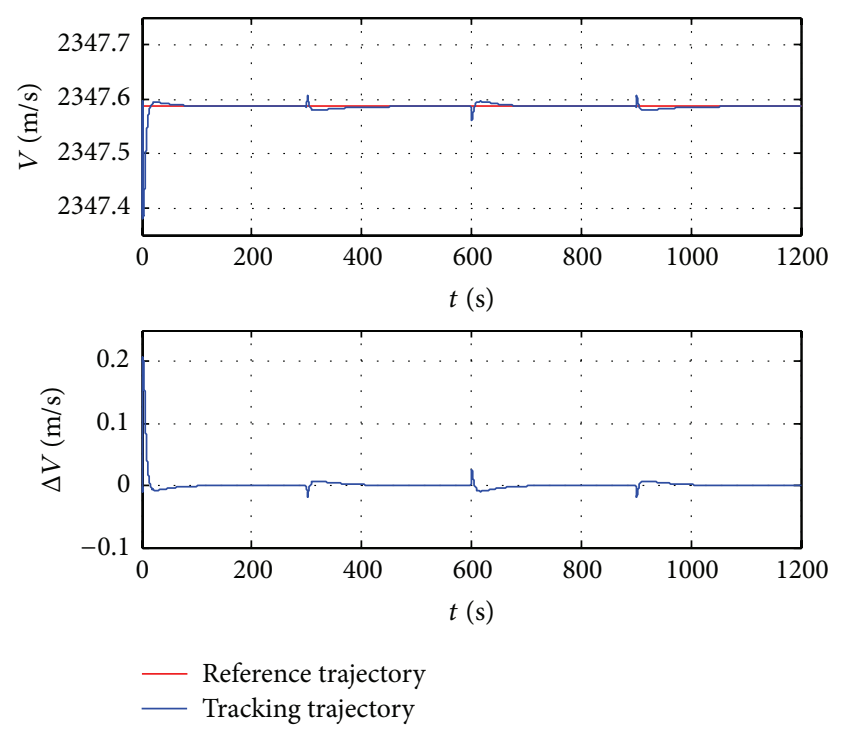

FIGURE 2: Response curves of velocity and velocity tracking error (Case 1).
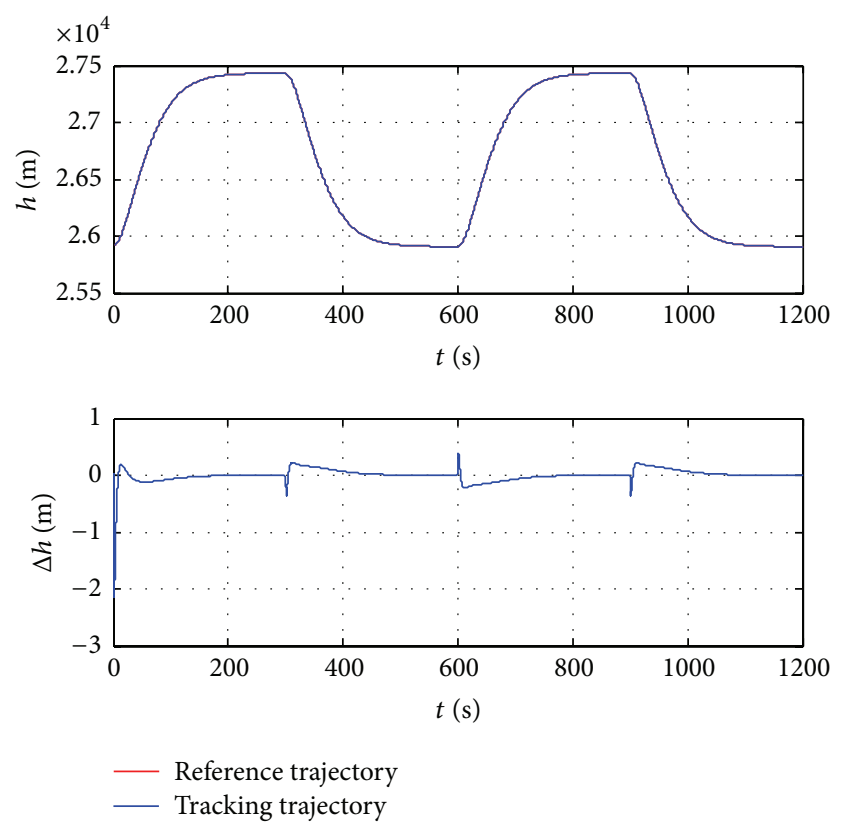

FIGURE 3: Response curves of altitude and altitude tracking error (Case 1).

$\theta_{f_{V}}^{M}=20, \theta_{g_{V}}^{M}=50, \theta_{f_{\gamma}}^{M}=0.01, \theta_{g_{\gamma}}^{M}=0.2, \theta_{f_{Q}}^{M}=5$, and $\theta_{g_{\mathrm{Q}}}^{M}=10$.

4.1. Trajectory Tracking Simulation without External Disturbance. To show efficiency of the proposed control law, the following two cases are proposed.

Case 1. The track height command is a square wave signal, whose amplitude $\Delta h_{c}=1524 \mathrm{~m}$ and period is $600 \mathrm{~s}$. 

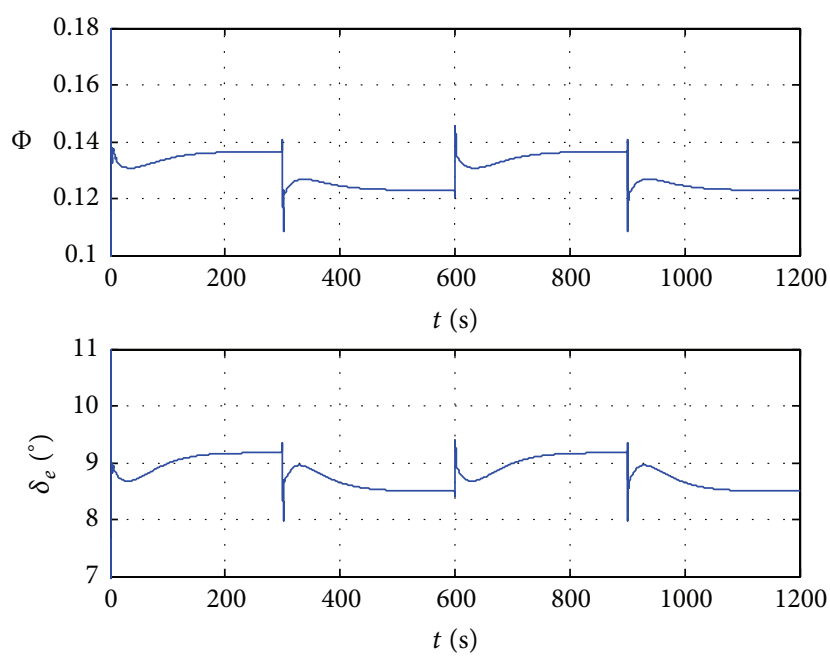

FIGURE 4: Time history of fuel equivalence ratio and elevator deflection (Case 1)
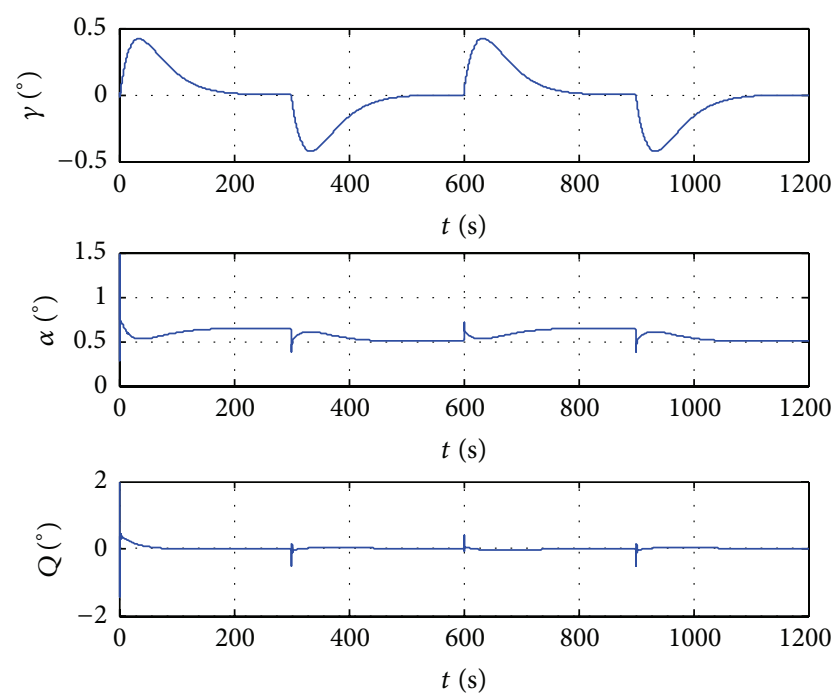

FIGURE 5: Response curves of flight path angle, attack angle, and pitch rate (Case 1$)$.

Case 2. The track height command is a square wave signal, whose amplitude $\Delta h_{c}=3048 \mathrm{~m}$ and period is $600 \mathrm{~s}$.

Time responses of velocity, altitude, flight path angle, attack angle, pitch rate, fuel equivalence ratio, elevator deflection, and flexible states in two cases are given in Figures 2-11.

Time responses to altitude step change in Case 1 are presented in Figures 2-6. As shown in Figures 2 and 3, the velocity and altitude can track their commands timely and without any steady tracking errors. The control inputs and attitude angles are smooth and within their bounds, just as shown in Figures 4 and 5. The flexible states can reach equilibrium state, which can be observed from Figure 6.

Time responses to altitude step change in Case 2 are presented in Figures 7-11. It can be seen from Figure 9 that the saturation occurs in elevator deflection during simulation,
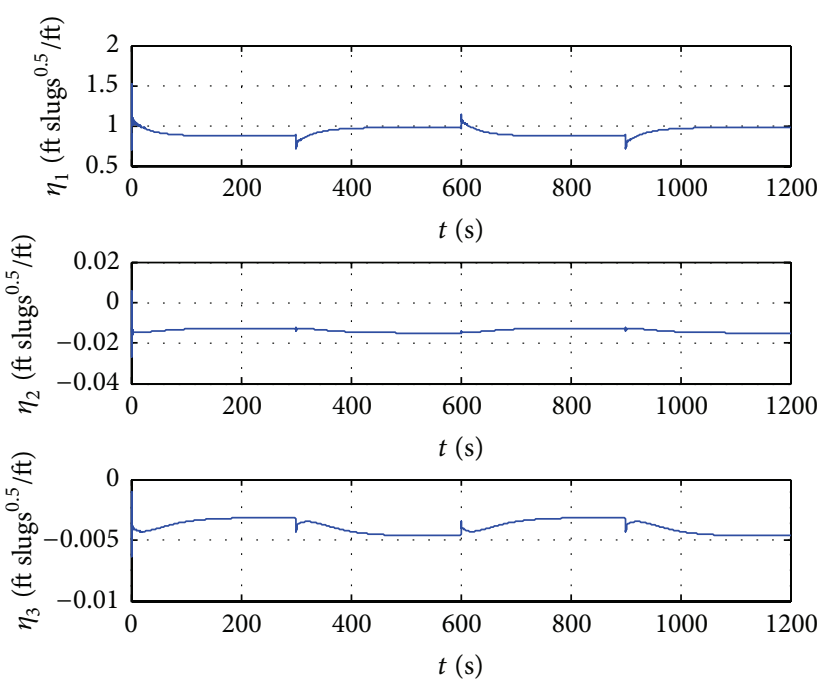

Figure 6: The flexible states (Case 1).
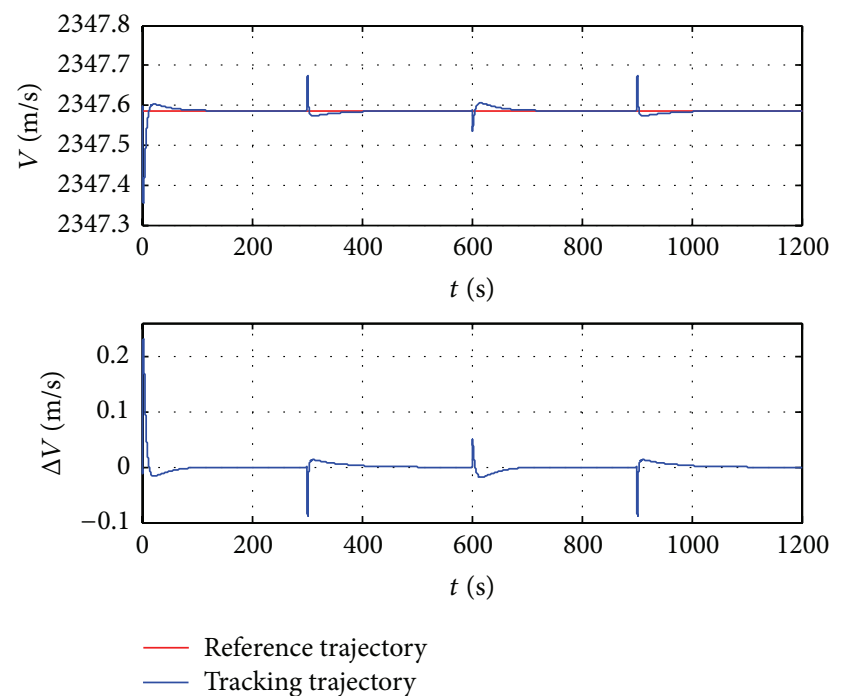

FIGURE 7: Response curves of velocity and velocity tracking error (Case 2).

but the controller still provides a good track characteristic according to Figures 7-10. Furthermore, the flexible states also can maintain stability, which can be observed from Figure 11.

4.2. Trajectory Tracking Simulation with External Disturbance. To illustrate the robustness to external disturbance of the control laws developed herein, two vertical wind disturbances whose amplitudes are $17 \mathrm{~m} / \mathrm{s}$ and $14 \mathrm{~m} / \mathrm{s}$ are added to the control system in $100 \mathrm{~s}$ and $300 \mathrm{~s}$, respectively, and the sustaining time of wind disturbance is $10 \mathrm{~s}$. The additional attack angle with wind disturbance can be expressed as

$$
\begin{aligned}
& \alpha_{u}=\arctan \frac{u \sin \gamma}{V+u \cos \gamma}, \\
& \alpha_{w}=\arctan \frac{w \cos \gamma}{V-w \sin \gamma},
\end{aligned}
$$



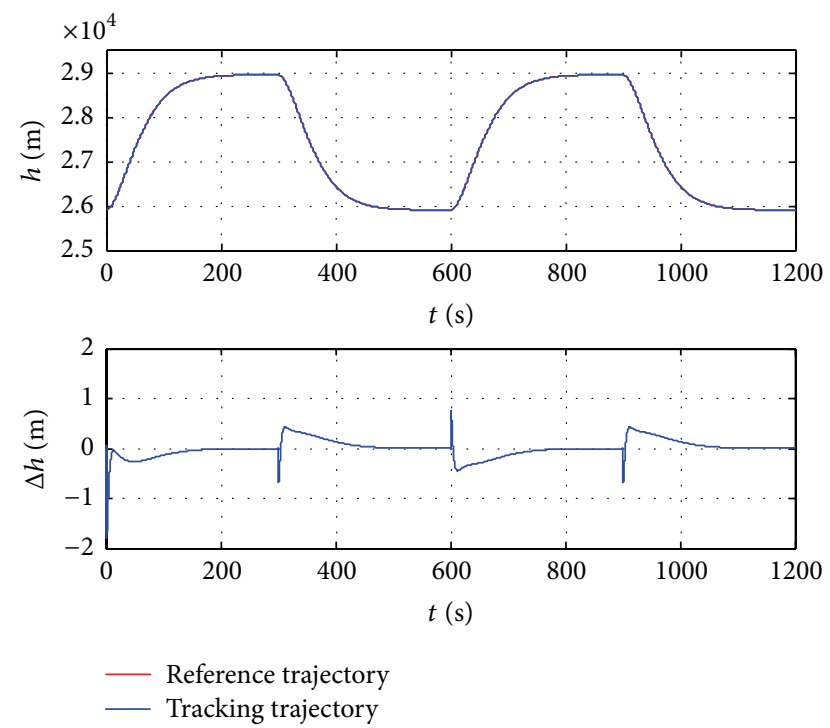

FIGURE 8: Response curves of altitude and altitude tracking error (Case 2).
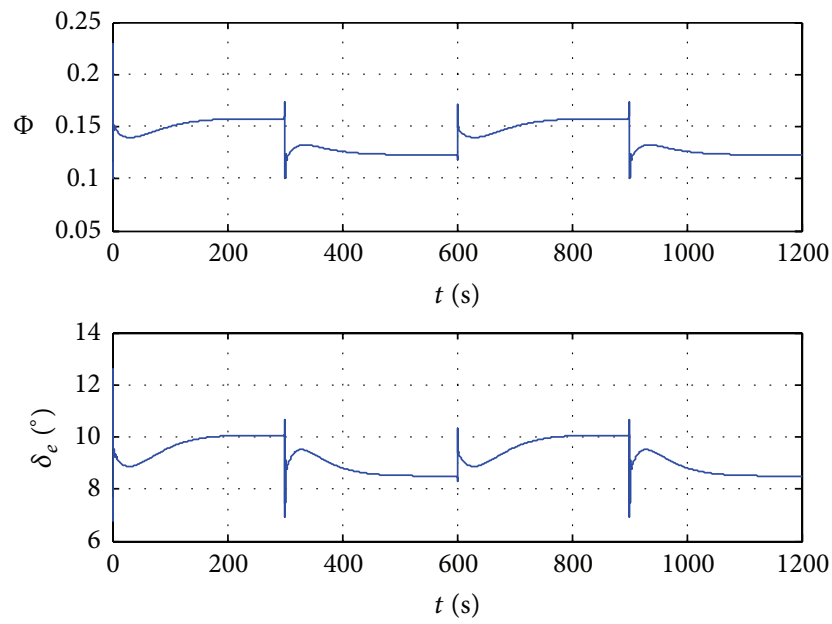

FIGURE 9: Time history of fuel equivalence ratio and elevator deflection (Case 2).

where $\alpha_{u}$ and $\alpha_{w}$ are horizontal additional attack angle and vertical additional attack angle, respectively; $u$ and $w$ are level wind speed and vertical wind speed, respectively.

The aircraft is commanded to perform a cruising flight mission that the initial height is $25908 \mathrm{~m}$ and dynamic pressure is $95760 \mathrm{~Pa}$. When the simulation time $t \leq 215 \mathrm{~s}$, altitude follows the step command with $50 \mathrm{ft} / \mathrm{s}$; when the simulation time $215 \mathrm{~s} \leq t \leq 355 \mathrm{~s}$, altitude follows the step command with $139 \mathrm{ft} / \mathrm{s}$.

The simulation results are illustrated in Figures 12-15. It can be seen from Figure 14 that the saturation occurs in elevator deflection with wind disturbance in $100 \mathrm{~s}$ and $300 \mathrm{~s}$, but the controller still provides a good track characteristic according to Figures 12-15, which indicate that the proposed adaptive neural back-stepping controller has a good
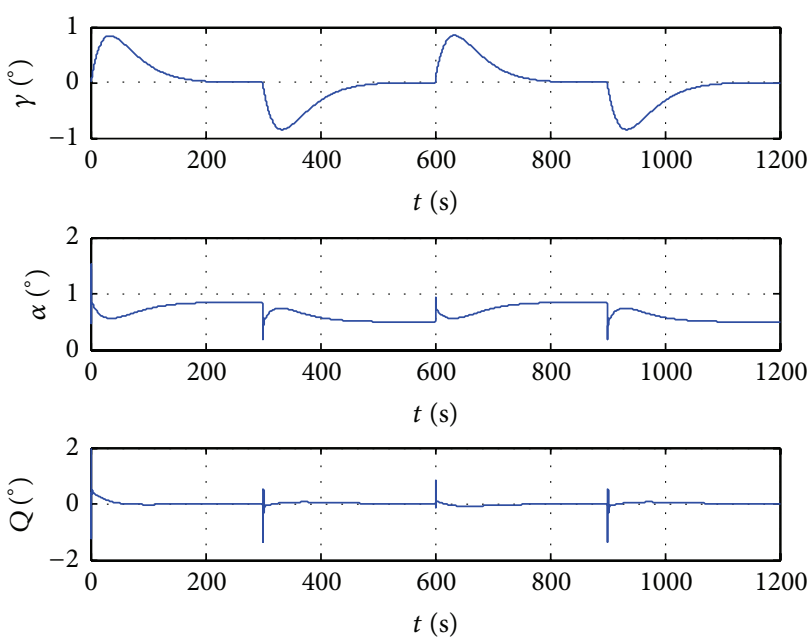

FIGURE 10: Response curves of flight path angle, attack angle, and pitch rate (Case 2).
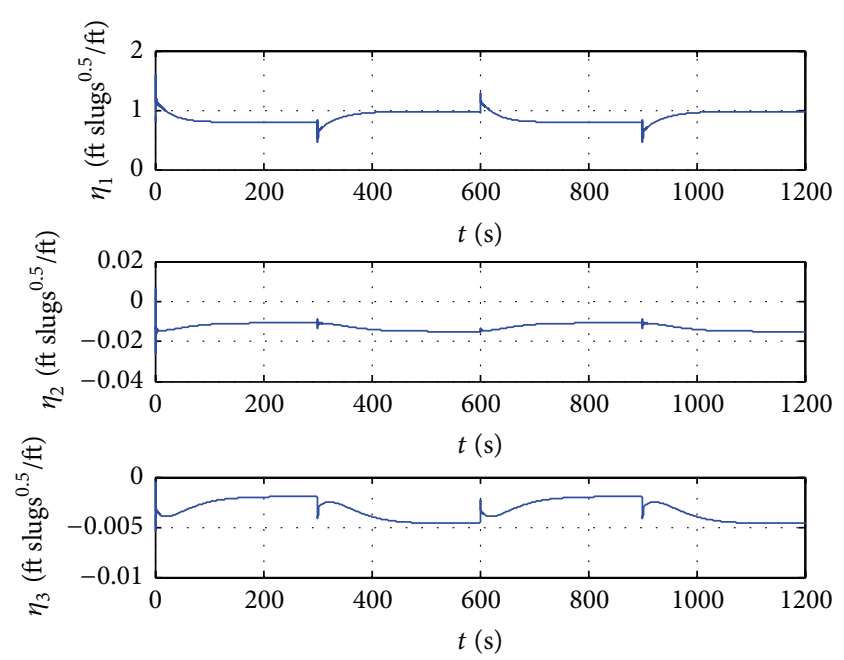

FIgure 11: The flexible states (Case 2).

performance on disposing input constraint under external disturbance.

\section{Conclusions}

An adaptive neural back-stepping control design is presented in this paper for hypersonic vehicle with input constraint and uncertain aerodynamic parameters. To reduce the complexity of the controller design, the nonlinear model is divided into two subsystems, and the adaptive neural back-stepping controllers are developed for them, respectively. The neural network based radial basis function is designed to approximate the unknown system nonlinearity, which needs less knowledge of dynamic model. In order to eliminate the problem of "explosion of term," a second-order reference model is designed for the precise estimation of the derivatives of virtual control laws. Expressly, the auxiliary systems are constructed to eliminate the saturation effect, where a novel 

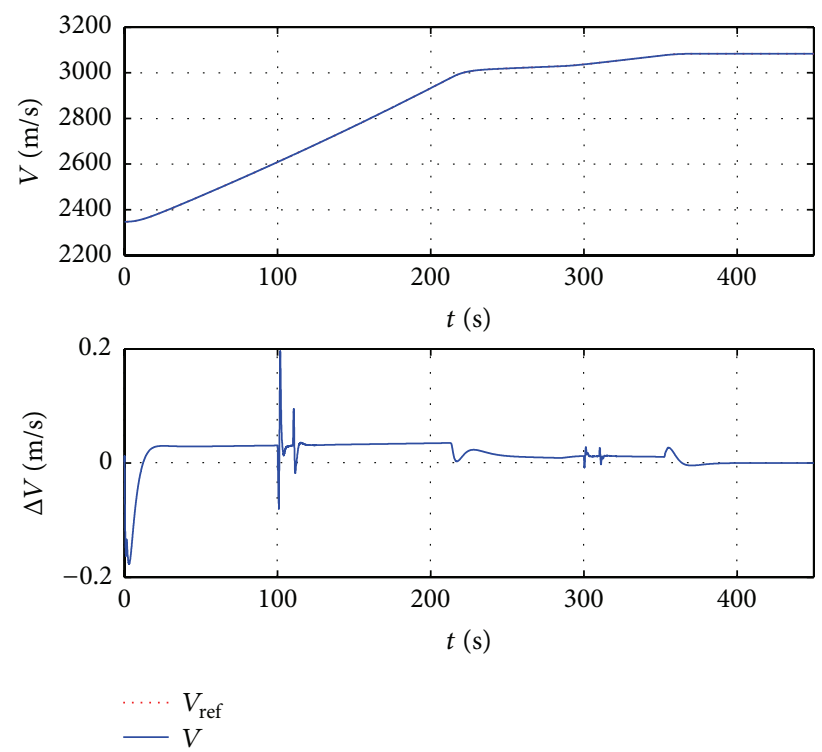

FIGURE 12: Response curves of velocity and velocity tracking error.
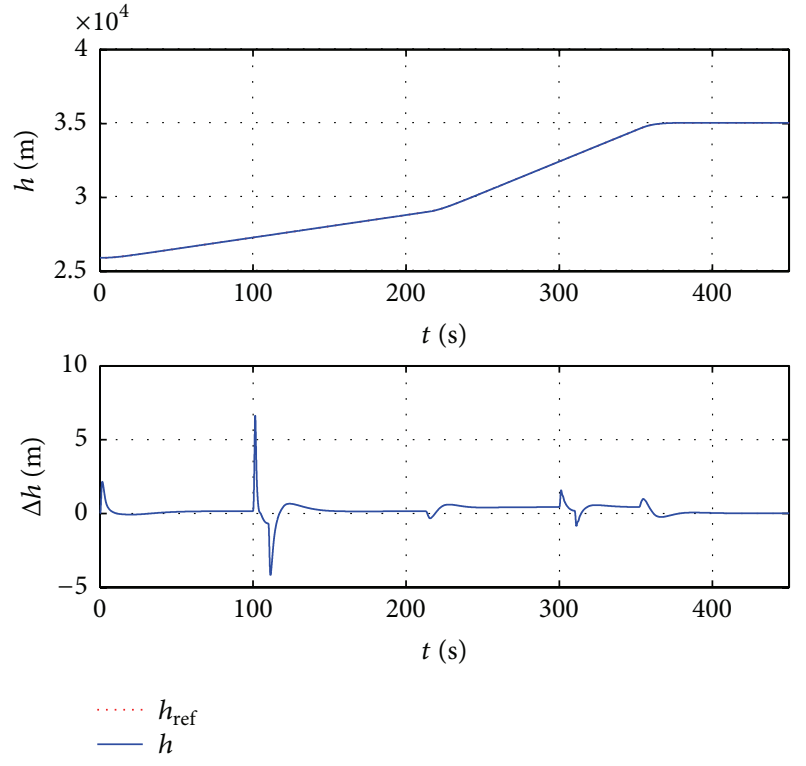

FIGURE 13: Response curves of altitude and altitude tracking error.

auxiliary error compensation scheme is proposed. A comprehensive Lyapunov based stability analysis is provided to show that the control design achieves ultimate boundedness tracking for the hypersonic vehicle. Simulation results demonstrate that the proposed control strategy can provide satisfactory velocity and altitude tracking performance with input constraint and uncertain aerodynamic parameters.

\section{Conflict of Interests}

The authors declare that there is no conflict of interests regarding the publication of this paper.
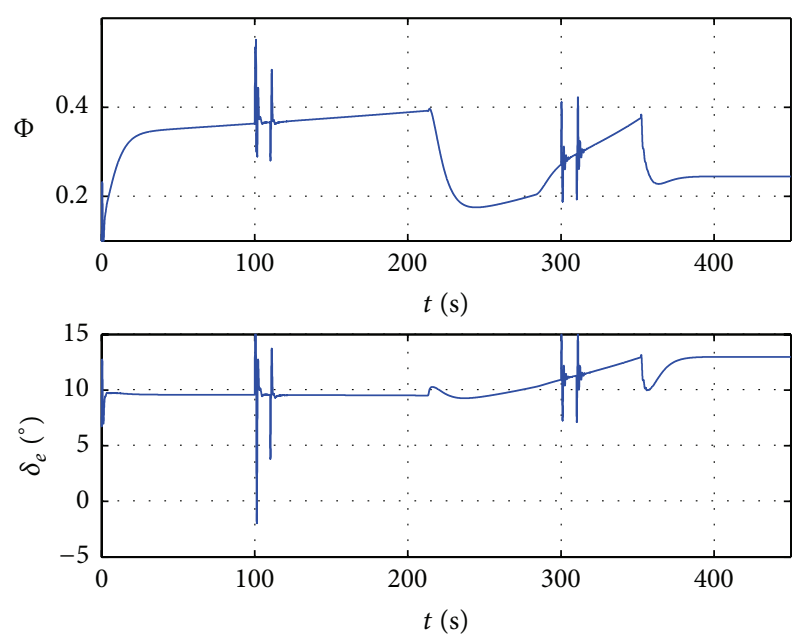

FIgURE 14: Time history of fuel equivalence ratio and elevator deflection.
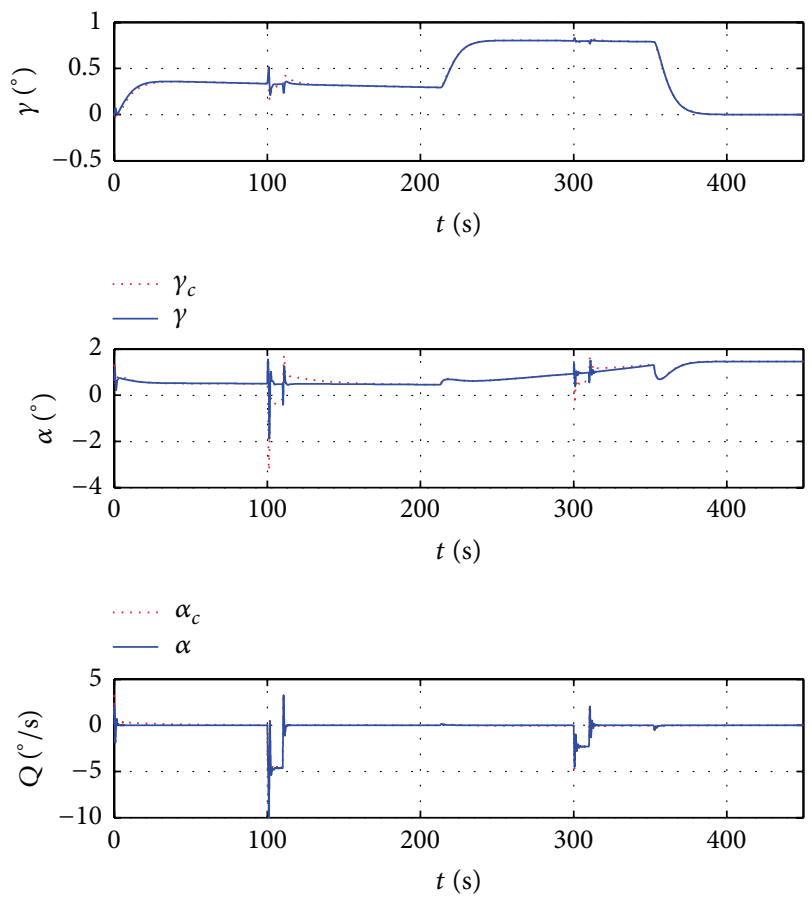

$\begin{array}{ll}\cdots & Q_{c} \\ - & Q\end{array}$

FIGURE 15: Response curves of flight path angle, attack angle, and pitch rate.

\section{Acknowledgment}

This work was supported by the Aeronautical Science Foundation of China (Grant no. 20120916006).

\section{References}

[1] Q. Zong, Y. Ji, F. Zeng, and H. Liu, "Output feedback backstepping control for a generic Hypersonic Vehicle via small-gain 
theorem," Aerospace Science and Technology, vol. 23, no. 1, pp. 409-417, 2012.

[2] Q. Zong, F. Wang, B. Tian, and R. Su, "Robust adaptive dynamic surface control design for a flexible air-breathing hypersonic vehicle with input constraint and uncertainty," Nonlinear Dynamics, vol. 78, no. 1, pp. 289-315, 2014.

[3] J. Wang, Q. Zong, R. Su, and B. Tian, "Continuous high order sliding mode controller design for a flexible air-breathing hypersonic vehicle," ISA Transactions, vol. 53, no. 3, pp. 690698, 2014

[4] C. I. Marrison and R. F. Stengel, "Design of robust control systems for a hypersonic aircraft," Journal of Guidance, Control, and Dynamics, vol. 21, no. 1, pp. 58-63, 1998.

[5] K. P. Groves, Modelling, Simulation, and Control Design of an Air-breathing Hypersonic Vehicle, Ohio State University, Columbus, Ohio, USA, 2005.

[6] P. V. Kokotovic, "The joy of feedback: nonlinear and adaptive," IEEE Control Systems, vol. 12, no. 3, pp. 7-17, 1992.

[7] T. Lee and Y. Kim, "Nonlinear adaptive flight control using backstepping and neural networks controller," Journal of Guidance, Control, and Dynamics, vol. 24, no. 4, pp. 675-682, 2001.

[8] X. Bu, X. Wu, R. Zhang, Z. Ma, and J. Huang, “Tracking differentiator design for the robust backstepping control of a flexible air-breathing hypersonic vehicle," Journal of the Franklin Institute. Engineering and Applied Mathematics, vol. 352, no. 4, pp. 1739-1765, 2015.

[9] X. Bu, X. Wu, Z. Ma, and R. Zhang, "Nonsingular direct neural control of air-breathing hypersonic vehicle via back-stepping," Neurocomputing, vol. 153, no. 1, pp. 164-173, 2015.

[10] D.-X. Gao, Z.-Q. Sun, and J.-H. Liu, "Dynamic inversion control for a class of pure-feedback systems," Asian Journal of Control, vol. 14, no. 2, pp. 605-611, 2012.

[11] D. Gao, Z. Sun, and B. Xu, "Fuzzy adaptive control for purefeedback system via time scale separation," International Journal of Control, Automation and Systems, vol. 11, no. 1, pp. 147-158, 2013.

[12] M. Sharma, J. A. Farrell, M. Polycarpou et al., "Back-stepping flight control using on-line function approximation," in Proceedings of the AIAA Guidance, Navigation, and Control Conference, Chicago, Ill, USA, August 2009.

[13] L. Fiorentini, Nonlinear adaptive controller design for airbreathing hypersonic vehicles [M.S. thesis], The Ohio State University, Columbus, Ohio, USA, 2010.

[14] B. Xu, F. Sun, H. Liu, and J. Ren, "Adaptive Kriging controller design for hypersonic flight vehicle via back-stepping," IET Control Theory \& Applications, vol. 6, no. 4, pp. 487-497, 2012.

[15] X. Bu, X. Wu, Y. Chen, and R. Bai, "Nonlinear-disturbanceobserver-based sliding mode backstepping control of hypersonic vehicles," Control Theory \& Applications, vol. 31, no. 11, pp. 1473-1479, 2014.

[16] X. W. Bu, X. Y. Wu, R. Zhang, and F. Zhu, "Design of a hyperbolic-sine-based nonlinear tracking differentiator," Journal of Xian Jiaotong University, vol. 49, no. 1, pp. 107-111, 2015.

[17] X. Bu, X. Wu, Z. Ma, and Y. Zhong, "Design of a modified arctangent-based tracking differentiator," Journal of Shanghai Jiaotong University, vol. 49, no. 2, pp. 164-168, 2015.

[18] X. Bu, X. Wu, Z. Ma, and R. Zhang, "Novel adaptive neural control of flexible air-breathing hypersonic vehicles based on sliding mode differentiator," Chinese Journal of Aeronautics, vol. 28, no. 4, pp. 1209-1216, 2015.
[19] N. O. Pérez-Arancibia, T.-C. Tsao, and J. S. Gibson, "Saturationinduced instability and its avoidance in adaptive control of hard disk drives," IEEE Transactions on Control Systems Technology, vol. 18, no. 2, pp. 368-382, 2010.

[20] Y.-Y. Cao and Z. L. Lin, "Robust stability analysis and fuzzyscheduling control for nonlinear systems subject to actuator saturation," IEEE Transactions on Fuzzy Systems, vol. 11, no. 1, pp. 57-67, 2003.

[21] M. Chen, S. S. Ge, B. V. E. How, and Y. S. Choo, "Robust adaptive position mooring control for marine vessels," IEEE Transactions on Control Systems Technology, vol. 21, no. 2, pp. 395-409, 2013.

[22] M. Chen, S. S. Ge, and B. V. E. How, "Robust adaptive neural network control for a class of uncertain MIMO nonlinear systems with input nonlinearities," IEEE Transactions on Neural Networks, vol. 21, no. 5, pp. 796-812, 2010.

[23] D. Dai, T. Hu, A. R. Teel, and L. Zaccarian, "Output feedback synthesis for sampled-data system with input saturation," in Proceedings of the American Control Conference (ACC '10), pp. 1797-1802, IEEE, Baltimore, Md, USA, July 2010.

[24] K. Y. Volyanskyy, W. M. Haddad, and J. M. Bailey, "Neuroadaptive output feedback control for nonlinear nonnegative dynamical systems with actuator amplitude and integral constraints," in Proceedings of American Control Conference (ACC '09), pp. 4494-4499, St. Louis, Mo, USA, June 2009.

[25] J. Zhou, Z. T. Liu, H. Y. Su, and C. Wen, "Robust adaptive control of uncertain nonlinear systems in the presence of input saturation and external disturbance," IEEE Transactions on Automatic Control, vol. 56, no. 7, pp. 1672-1678, 2011.

[26] J. H. Huang, C. Y. Wen, W. Wang, and Z.-P. Jiang, "Adaptive stabilization and tracking control of a nonholonomic mobile robot with input saturation and disturbance," Systems and Control Letters, vol. 62, no. 3, pp. 234-241, 2013.

[27] M. A. Bolender and D. B. Doman, "Nonlinear longitudinal dynamical model of an air-breathing hypersonic vehicle," Journal of Spacecraft and Rockets, vol. 44, no. 2, pp. 374-387, 2007.

[28] Q. Zong, F. Wang, B. Tian, and R. Su, "Robust adaptive approximate backstepping control of a flexible air-breathing hypersonic vehicle with input constraint and uncertainty," Proceedings of the Institution of Mechanical Engineers I, vol. 228, no. 7, pp. 521-539, 2014.

[29] A. Serrani, A. M. Zinnecker, L. Fiorentini, M. A. Bolender, and D. B. Doman, "Integrated adaptive guidance and control of constrained nonlinear air-breathing hypersonic vehicle models," in Proceedings of the American Control Conference (ACC '09), pp. 3172-3177, IEEE, St. Louis, Mo, USA, June 2009.

[30] A. A. Rodriguez, J. J. Dickeson, O. Cifdaloz et al., "Modeling and control of scramjet-powered hypersonic vehicles: challenges, trends, and tradeoffs," in Proceedings of the AIAA Guidance, Navigation and Control Conference and Exhibit, Honolulu, Hawaii, USA, August 2008.

[31] M. Chen, S. S. Ge, and B. V. E. How, "Robust adaptive neural network control for a class of uncertain MIMO nonlinear systems with input nonlinearities," IEEE Transactions on Neural Networks, vol. 21, no. 5, pp. 796-812, 2010.

[32] M. Chen, S. S. Ge, and B. B. Ren, "Adaptive tracking control of uncertain MIMO nonlinear systems with input constraints," Automatica, vol. 47, no. 3, pp. 452-465, 2011. 


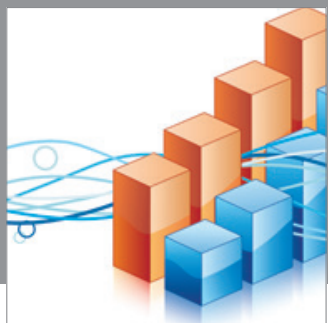

Advances in

Operations Research

mansans

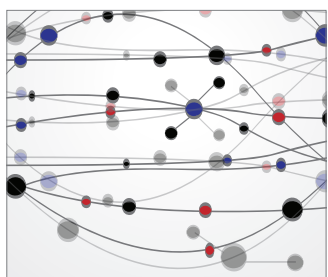

The Scientific World Journal
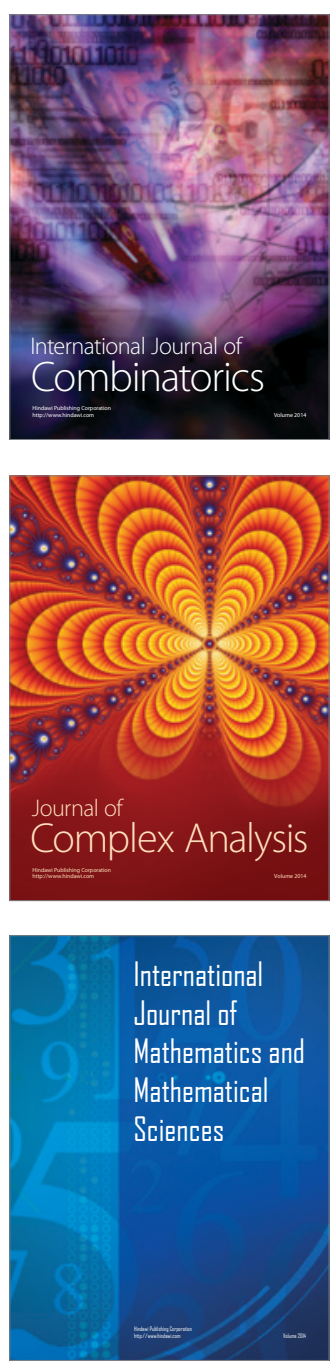
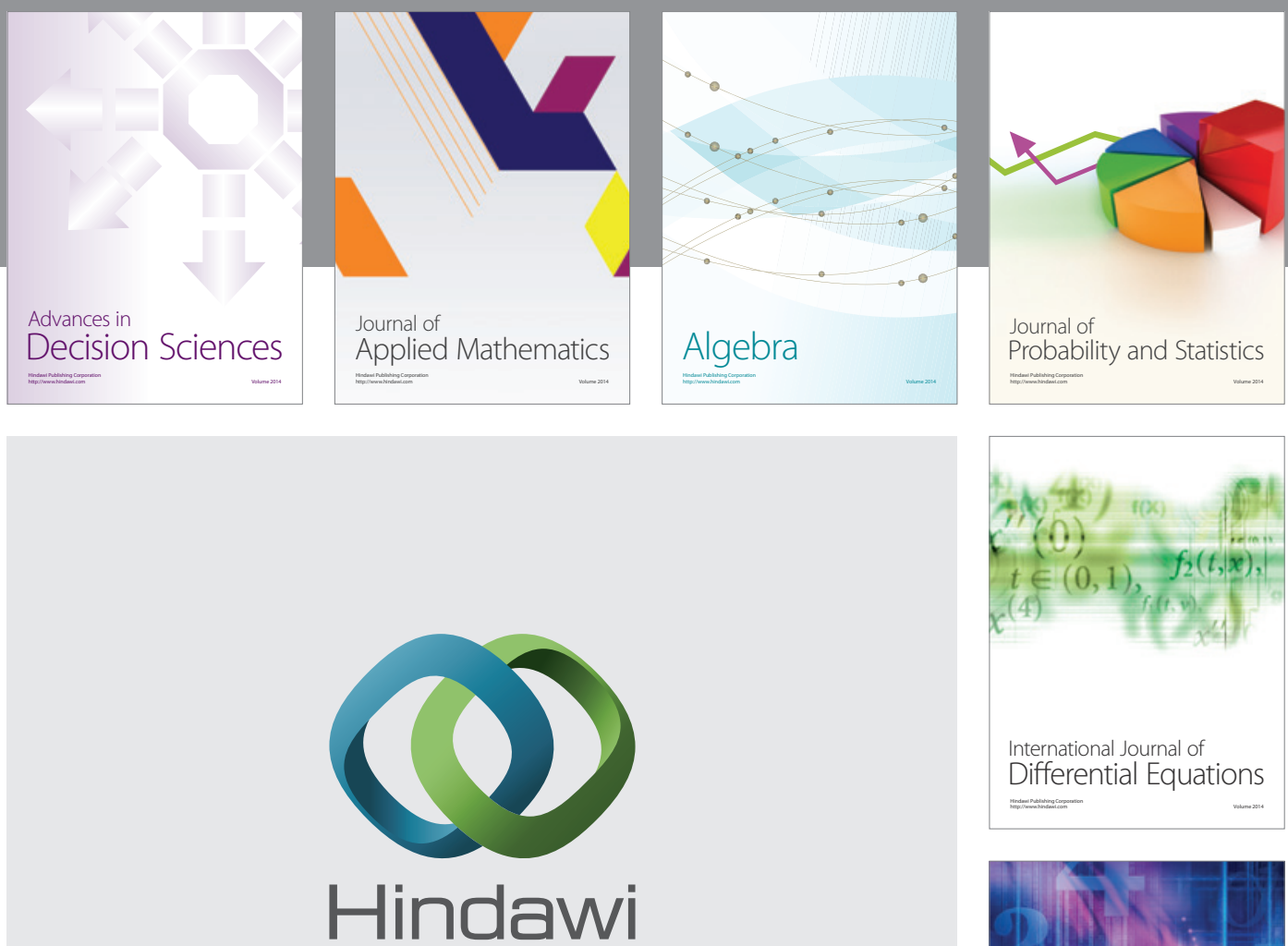

Submit your manuscripts at http://www.hindawi.com
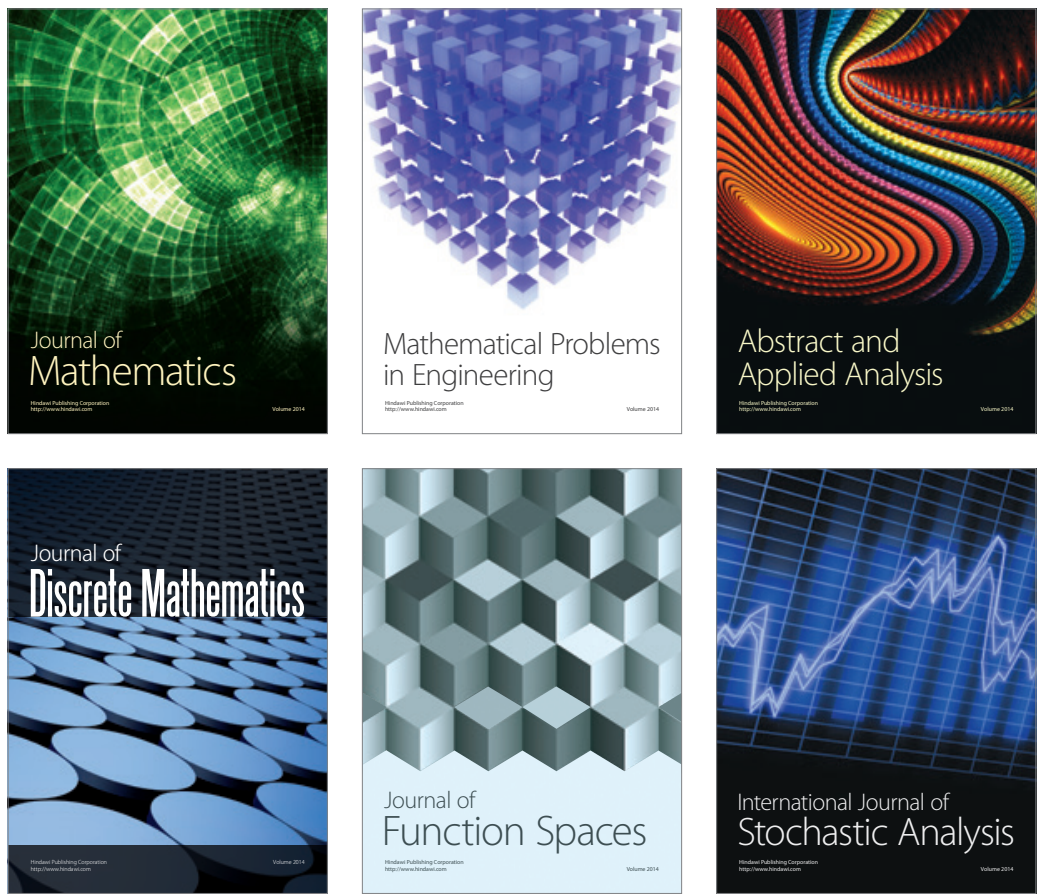

Journal of

Function Spaces

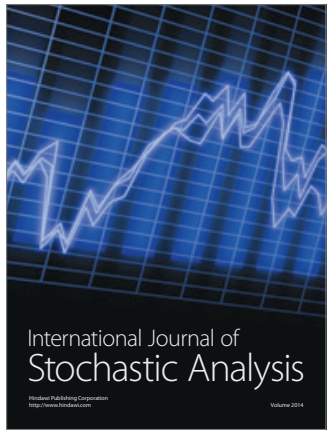

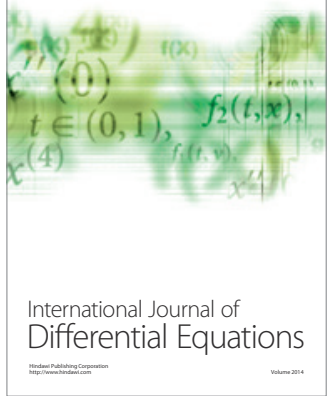
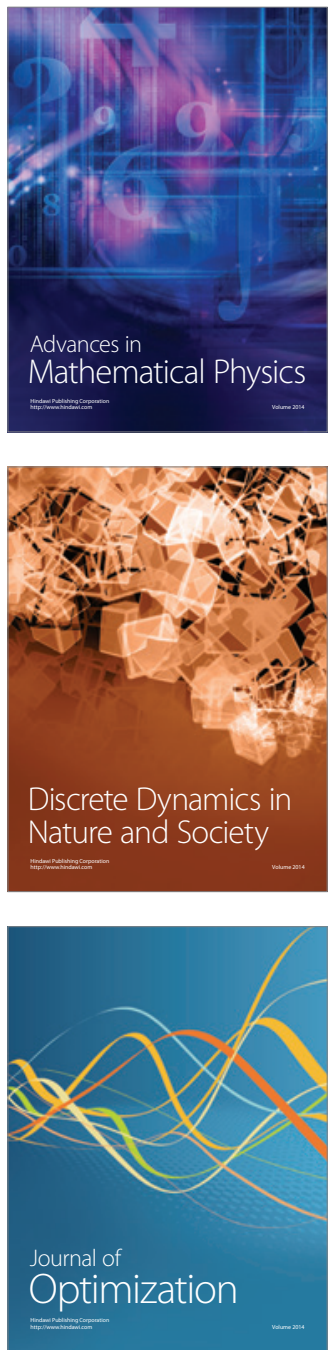